\title{
Comparative Proteomics Reveals the Potential Targets of BcNoxR, a Putative Regulatory Subunit of NADPH Oxidase of Botrytis cinerea
}

\author{
Hua $\mathrm{Li},{ }^{1,2}$ Zhanquan Zhang, ${ }^{1}$ Chang He, ${ }^{1,2}$ Guozheng Qin, ${ }^{1}$ and Shiping Tian ${ }^{1,2}$ \\ ${ }^{1}$ Key Laboratory of Plant Resources, Institute of Botany, Chinese Academy of Sciences, Beijing 100093, China; and \\ ${ }^{2}$ University of Chinese Academy of Sciences, Beijing 100049, China \\ Accepted 23 November 2016.
}

\begin{abstract}
The NADPH oxidase (NOX) complex has been shown to play a crucial role in stress response and in the virulence of various fungal pathogens. The underlying molecular mechanisms of NOX, however, remain largely unknown. In the present study, a comparative proteomic analysis compared changes in protein abundance in wild-type Botrytis cinerea and $\Delta b c n o x R$ mutants in which the regulatory subunit of NOX was deleted. The $\Delta b c n o x R$ mutants exhibited reduced growth, sporulation, and impaired virulence. A total of 60 proteins, representing 49 individual genes, were identified in $\Delta$ bcnox $R$ mutants that exhibited significant differences in abundance relative to wild-type. Reverse transcription-quantitative polymerase chain reaction analysis demonstrated that the differences in transcript levels for 36 of the genes encoding the identified proteins were in agreement with the proteomic analysis, while the remainder exhibited reverse levels. Functional analysis of four proteins that decreased abundance in the $\Delta b c n o x R$ mutants indicated that 6-phosphogluconate dehydrogenase (BcPGD) played a role in the growth and sporulation of $B$. cinerea. The $\Delta$ bcpgd mutants also displayed impaired virulence on various hosts, such as apple, strawberry, and tomato fruit. These results suggest that NOX can influence the expression of BcPGD, which has an impact on growth, sporulation, and virulence of $B$. cinerea.
\end{abstract}

Reactive oxygen species (ROS) are highly reactive molecules, such as superoxide anion $\left(\cdot \mathrm{O}_{2}^{-}\right)$, hydroxyl radical $(\cdot \mathrm{OH})$, and hydrogen peroxide $\left(\mathrm{H}_{2} \mathrm{O}_{2}\right)$. ROS can be generated as a byproduct of metabolism in mitochondria, peroxisomes, cytochrome P-450, and other cellular components. NADPH oxidase (NOX), a multisubunit complex, was the first enzyme system identified that generates ROS as its primary function rather than as a byproduct. NOX generates superoxide through a one-electron reduction of molecular oxygen, using electrons supplied by NADPH (Takemoto et al. 2011). Superoxide can be directly converted to $\mathrm{H}_{2} \mathrm{O}_{2}$ via superoxide dismutase, which can then be further converted to other forms of ROS. NOX is composed of catalytic subunits, regulatory subunits, and other functional

Hua Li and Zhanquan Zhang contributed equally to this work.

Corresponding author: S. Tian; E-mail: tsp@ibcas.ac.cn; Telephone: +86-10-6283-6559; Fax: +86-10-8259-4675

*The $\boldsymbol{e}$-Xtra logo stands for "electronic extra" and indicates that seven supplementary figures and three supplementary tables are published online.

(c) 2016 The American Phytopathological Society proteins. NOX2 in humans, the most researched NOX, is a complex of catalytic subunit gp $91^{\text {phox }}$, regulatory subunit $\mathrm{p} 67^{\text {phox }}$, and the functional proteins $\mathrm{p} 47^{\text {phox }}$, p40 ${ }^{\text {phox }}$, and Rac (Diekmann et al. 1994). The NOX complex is typically inactive until it is activated by the presence of a pathogen. When activated, the regulatory subunits and other functional proteins combine with the catalytic subunits. Activated NOX induces $\mathrm{K}^{+}$influx, giving rise to changes in $\mathrm{pH}$, which activates neutral proteases and causes the death of pathogens (Reeves et al. 2002). Plants are also capable of producing ROS through enzymes identified as respiratory burst oxidase homologs (RBOHs), which are homologs of NOX (Suzuki et al. 2011). Arabidopsis RBOHs have been reported to be required for plant defense response (Torres et al. 2002), symbiotic nodule functioning (Marino et al. 2011), and developmental processes (Müller et al. 2009).

In most fungi, two different subunits, NoxA and NoxB, have been identified. Both NoxA and NoxB are homologs of the human catalytic subunit gp91 $1^{\text {phox }}$ and possess the core NOX transmembrane and catalytic domains (Takemoto et al. 2007). NoxA and NoxB play an essential role in multicellular development. NoxA is essential for the development of sexual structures in Aspergillus nidulans and Neurospora crassa (Cano-Domínguez et al. 2008; Lara-Ortíz et al. 2003), while NoxB regulates ascospore germination in Neurospora crassa and Podospora anserina (Cano-Domínguez et al. 2008; Malagnac et al. 2004). Both BcNoxA and BcNoxB are responsible for the formation of sclerotia, however, neither is required for ascospore germination in B. cinerea (Segmüller et al. 2008). NoxA and NoxB are also essential for the development of penetration structures and hyphal extension during the infection of host plants. Dysfunction of NoxA or NoxB is responsible for the loss of virulence in Magnaporthe oryzae, due to the inability of the pathogen to develop a normal penetration peg (Egan et al. 2007). In B. cinerea, BcNoxB plays an essential role in the formation of the penetration structure, while $\mathrm{BcNoxA}$ is required for hyphal growth after infection has occurred (Segmüller et al. 2008). The catalytic function of both NoxA and NoxB are regulated by NoxR, which is a homolog of the human regulatory subunit $\mathrm{p} 67^{\text {phox }}$. Deletion of NoxR in A. nidulans results in a deficiency in the formation of cleistothecia, a result that is similar to the phenotype of the NoxA mutant (Scott and Eaton 2008; Semighini and Harris 2008). The $\Delta$ bcnoxR mutant in $B$. cinerea exhibits aberrant penetration and colonization of plant tissues, a phenotype that is identical to that of the $\Delta$ bcnoxA/B double mutant (Segmüller et al. 2008). It is speculated that $\mathrm{BcNoxR}$ interact with $\mathrm{BcNoxA} / \mathrm{B}$ through adaptor proteins like $\mathrm{BcNoxD}$, since no direct interaction of $\mathrm{BcNoxR}$ and $\mathrm{BcNoxA} / \mathrm{B}$ has been confirmed (Siegmund et al. 2015). Additionally, BemA 
and Cdc24, which have been reported to interact with NoxR in Epichloe festucae, are involved in polarity establishment in fungal cells, which has a direct impact on hyphal growth (Kayano et al. 2013). Although NOX has been shown to play multiple roles in fungi, little is known about the molecular mechanisms by which NOX functions in these biological processes.

$B$. cinerea is a necrotrophic, phytopathogenic fungus that infects over 200 plant species (Weiberg et al. 2013). In the past decade, $B$. cinerea has been extensively used as a model system for studying the virulence of aggressive fungal pathogens due to its broad host range and the extensive economic losses that it causes (An et al. 2016; Li et al. 2012). Studies utilizing targeted mutagenesis of $B$. cinerea have become more frequent in the literature, in which it has been used to examine the role of different genes of interest (An et al. 2015; Kars et al. 2005). The release and improvement of genomic information on $B$. cinerea B05.10 has provided the foundation of information that has led to an increased understanding of this devastating pathogen, enabling the use of a variety of techniques, such as genomics, transcriptomics, and proteomics (Amselem et al. 2011; FernándezAcero et al. 2006).

In the present study, a comparative proteomic approach was used in $B$. cinerea to investigate the underlying mechanisms associated with the regulation of virulence by NOX. A total of 60 proteins, representing 49 individual genes, were identified in $\Delta b c n o x R$ mutants that exhibited significant differences in abundance relative to the wild type (WT). Reverse transcriptionquantitative polymerase chain reaction (RT-qPCR) analysis demonstrated that approximately $75 \%$ of the differentially expressed genes exhibit corresponding transcription and protein levels, although the specific relative abundance levels vary. Four of the differentially expressed proteins were selected for further functional studies by constructing deletion mutants. Results indicate that 6-phosphogluconate dehydrogenase (BcPGD), whose abundance decreased in the $\Delta b c n o x R$ mutant, plays a functional role in determining the virulence of $B$. cinerea.

\section{RESULTS}

\section{Bcnox $R$ is required for virulence.}

In order to investigate the role of bcnoxR in the virulence of $B$. cinerea, deletion mutants were generated by replacing the bcnoxR open reading frame (ORF) with a hygromycin $\mathrm{B}$ phosphotransferase (hph) marker by transforming protoplasts of the WT strain B05.10. Eight independent transformants were obtained by screening the putative transformants on selection media supplemented with hygromycin. Transformants were further confirmed by PCR. Three homozygous strains $\Delta$ bcnox $R 1, \Delta$ bcnox $R 2$, and $\Delta$ bcnox $R 9$, were further confirmed by Southern blot analysis using cells derived from single-spore isolation (Supplementary Fig. S1). Deletion of bcnoxR resulted in only a slight reduction in the vegetative growth rate but a significant decrease in sporulation. The deletion mutants also failed to form any sclerotia, structures that are essential for sexual development; thus indicating that bcnoxR has a strong impact on sexual reproduction in $B$. cinerea.

The ability of WT and $\Delta b c n o x R$ mutants to form infection structures on the surface of cellophane was examined. Microscopic analysis indicated that both the WT and $\Delta$ bcnox $R$ strains could form appressoria within $10 \mathrm{~h}$ postinoculation (hpi). The ability of the WT and mutant strains to penetrate onion epidermis was also examined. Results indicated that hyphae arising from the appressoria of the WT were able to penetrate the onion epidermis but that hyphae originating from the appressoria of the $\Delta b c n o x R$ mutants simply grew along the surface of the onion epidermis (Supplementary Fig. S2).
To compare differences in the virulence of the mutants and WT, the different strains were inoculated into wounds in apple, strawberry, and tomato fruits. Deletion of bcnoxR significantly reduced the virulence of $B$. cinerea on all three kinds of fruit. Obvious lesions could be observed $72 \mathrm{~h}$ after inoculation in apple fruit wounds, in which the lesion diameter of the mutants was significantly smaller than the lesion size in WT (Fig. 1A and B). In contrast, strawberry fruits exhibited visible lesions by $36 \mathrm{hpi}$ and tomato fruits exhibited visible lesions $48 \mathrm{hpi}$ (Fig. $1 \mathrm{C}$ to F). Disease development was also assessed on detached tomato leaves. The reduced virulence of the mutants was even more evident on detached tomato leaves, especially at $48 \mathrm{hpi}$, when the WT exhibited a rapidly expanding lesion while the mutant strains had not produced a visible lesion at this time point (Fig. 1G and $\mathrm{H}$ ). The reduced virulence of the mutants on apple fruits and detached tomato leaves was restored by introducing the bcnoxR gene into the mutant strains (Supplementary Fig. S3). Collectively, these results suggest that bcnox $R$ is required for the virulence of $B$. cinerea.

\section{Protein expression is altered in the $\Delta b c n o x R$ mutant.}

$\Delta b c n o x R$ mutants are characterized by a reduced growth rate and decreased virulence as to the WT. In order to investigate the mechanism by which BcNoxR influences the virulence of $B$. cinerea, a comparative proteomic analysis was conducted using high-resolution two-dimensional (2D) gel electrophoresis. Proteins were extracted from the WT and one of the $\Delta$ bcnoxR mutants ( $\Delta$ bcnoxR 1$) 48 \mathrm{~h}$ after spores were cultured in liquid potato dextrose broth (PDB). Protein extracts were obtained using a phenol-based method that reduced the coextraction of contaminating compounds such as polysaccharides and lipids. The extracted total hyphal proteins were separated by isoelectric focusing on an immobilized linear $\mathrm{pH}$ gradient ranging from $\mathrm{pH} 4$ to 7 and, then, by sodium dodecyl sulfatepolyacrylamide gel electrophoresis (SDS-PAGE). Three biological replicates of the $\Delta b c n o x R$ and WT were analyzed, and representative gel images are shown in Figure 2A. Gel images were analyzed using Image Master 2D Elite software (GE Healthcare Bio-Sciences AB) and spots were scored only when they were detected in all three independent replicates. Approximately 1,300 protein spots were detected on each $2 \mathrm{D}$ gel after the exclusion of very faint spots and spots with undefined shapes and areas. A total of 74 spots were identified whose abundance was significantly different in the $\Delta b c n o x R$ mutant than in the WT by more than twofold according to a Student's $t$ test $(P<0.05)$. These spots were excised from the gels, were digested with trypsin, and were submitted to quadrupole-timeof-flight tandem mass spectrometry (Q-TOF MS/MS) analysis. MS/MS spectra were used for database searching in the Mascot search engine. A total of 60 protein spots, representing 49 individual genes, were successfully identified with Mowse scores greater than the threshold (Supplementary Table S1).

The identified proteins were classified into functional categories using blast2go software (Fig. 2B). Approximately a quarter of the differentially expressed proteins were related to stress response, such as heat shock 70-kDa protein 2 (spot 1), thiazole biosynthetic enzyme (spot 5), and thiamine synthase (spot 12). These results support the view that NOX functions in response to various environmental stresses in fungi (Lambeth and Neish 2014; Segmüller et al. 2008). Five of the differentially expressed proteins were associated with oxidation reduction, including delta-1-pyrroline-5-carboxylate dehydrogenase (spot 22), retinol dehydrogenase 8 protein (spot 57), peroxiredoxin PRX1 (spot 60), catalase protein (spot 67), and retinol dehydrogenase (spot 68). These results implied that NOX may influence fungal growth and virulence through affecting the abundance of a variety of redox proteins. 


\section{Deletion of bcnox $R$ affects the overall pattern} of protein expression.

Abundance data for the 60 identified proteins were clustered to characterize patterns of protein expression. Protein spots were clustered based on changes in spot intensity between the WT and $\Delta b c n o x R$ mutant, using the unweighted pair group method with an arithmetic mean (UPGMA) (Caraux and Pinloche 2005) (Fig. 3A). Two clusters of differentially abundant proteins were found. Cluster I (60\% of all spots) comprised 36 proteins whose abundance was higher in the $\Delta b c n o x R$ mutant. Approximately $27.78 \%$ of these proteins are associated with stress response, $16.67 \%$ are associated with carbohydrate
A

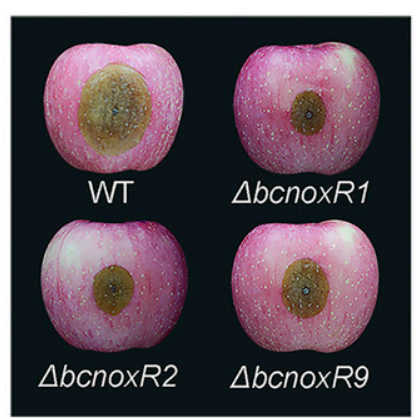

C

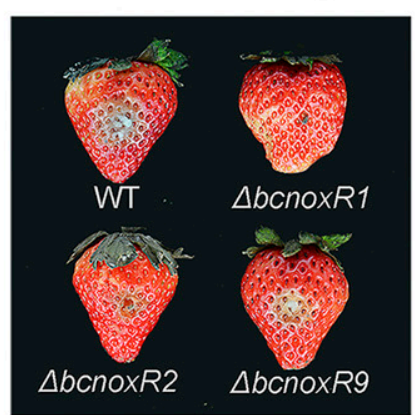

E

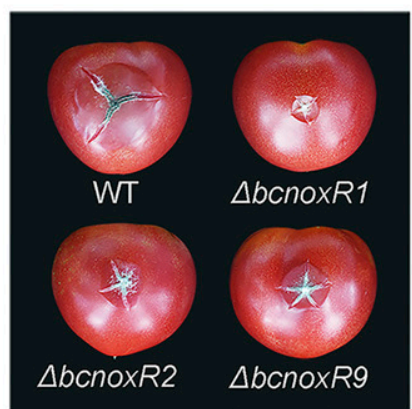

G

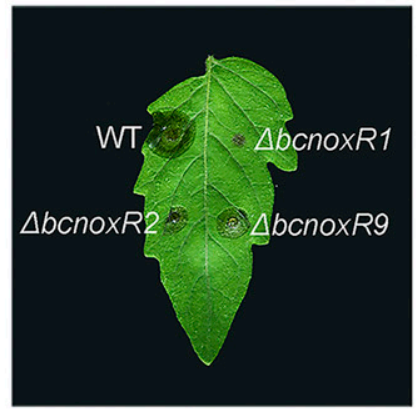

B

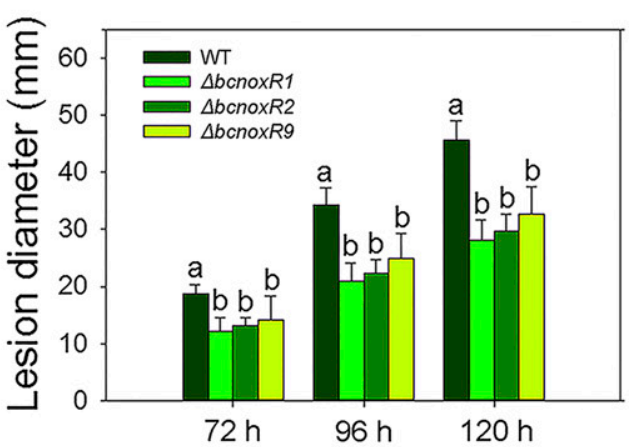

.

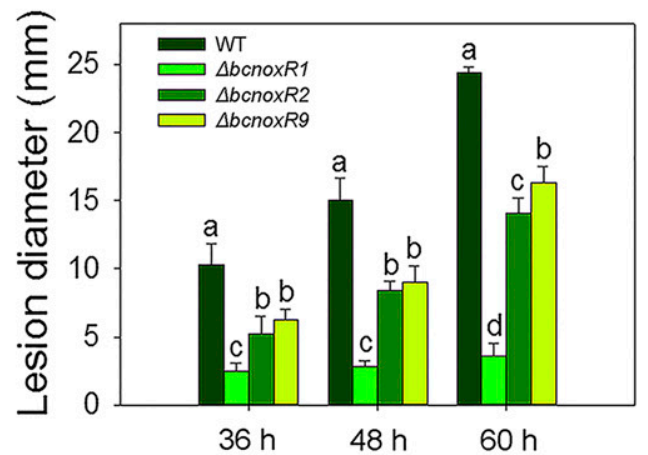

$\mathbf{F}$

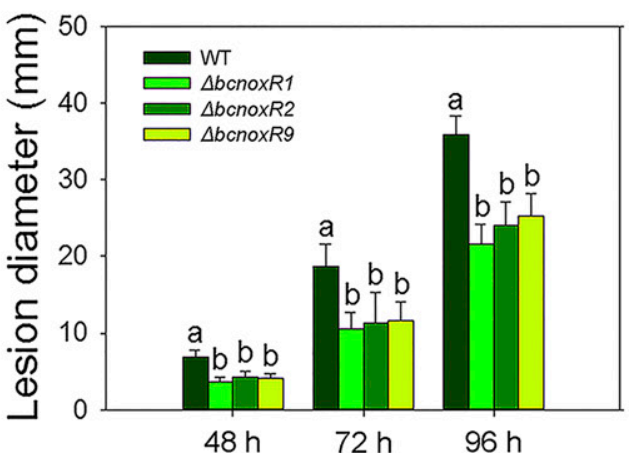

$\mathbf{H}$

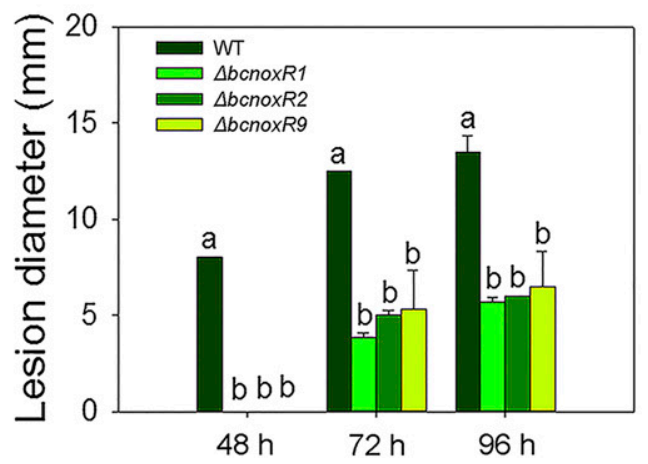

Fig. 1. $\Delta$ bcnoxR exhibits significantly reduced virulence on apple, strawberry, tomato fruits, and detached tomato leaves. A and B, Disease symptoms and statistical analysis of lesion diameter (mm) in wild type (WT), $\Delta$ bcnoxR1, $\Delta$ bcnoxR2, and $\Delta b c n o x R 9$ on apple fruits. Photos were taken $120 \mathrm{~h}$ after inoculation. $\mathbf{C}$ and D, Disease symptoms and statistical analysis of lesion diameter (mm) in WT, $\Delta b c n o x R 1, \Delta b c n o x R 2$, and $\Delta b c n o x R 9$ on strawberry fruits. Photos were taken $60 \mathrm{~h}$ after inoculation. $\mathbf{E}$ and $\mathbf{F}$, Disease symptoms and statistical analysis of lesion diameter (mm) in WT, $\Delta b c n o x R 1, \Delta b c n o x R 2$, and $\Delta b c n o x R 9$ on tomato fruits. Photos were taken $96 \mathrm{~h}$ after inoculation. $\mathbf{G}$ and $\mathbf{H}$, Disease symptoms and statistical analysis of lesion diameter (mm) in WT, $\Delta b c n o x R 1$, $\Delta$ bcnoxR2, and $\Delta$ bcnoxR 9 on detached tomato leaves. Photos were taken $72 \mathrm{~h}$ after inoculation. Data presented are the mean \pm standard deviation $(n=3)$. Columns with different letters at the same time are significantly different from each other, using the least significant difference test $(P<0.05)$. 
metabolism, and the remainding proteins are associated with translation, intracellular signaling, and other functional categories (Fig. 3B). Several proteins related to lipid metabolism, the Krebs cycle, amino saccharide catabolism, protein transport, glycolysis and gluconeogenesis, DNA methylation, and cytoskeleton were found exclusively in cluster I. Cluster II (40\% of all spots) comprised 24 proteins whose abundance was lower in the $\Delta$ bcnoxR mutant (Fig. 3C). These proteins are also mainly involved in stress response (approximately $25 \%$ of the spots), carbohydrate metabolism (approximately 20.83\%), and oxidation reduction (approximately 16.67\%). Proteins related to secondary metabolism, protein degradation, and resistance proteins were found exclusively in cluster II.

\section{Comparisons of gene expression}

in WT and the $\Delta$ bcnox $R$ mutants.

Sixty protein spots, representing 49 individual genes, exhibited significant differences in abundance in the $\Delta b c n o x R$ mutant, relative to the abundance observed in the WT. RT-qPCR was conducted to assay the expression of these 49 genes in transcript levels. RNAs were isolated from three independent biological replicates of WT and the $\Delta$ bcnoxR mutant. Gene sequences corresponding to the identified proteins were obtained from the EnsemblFungi Botrytis cinerea database. Results indicated that transcript levels of 36 of the genes (approximately $75 \%$ ) were in agreement with the protein abundance data, although the magnitude of the difference in transcript expression versus protein abundance varied (Fig. 4). Some genes exhibited smaller differences in transcript levels than in protein abundance, including heat shock protein 84 (spot 7), dnaK-type molecular chaperone $\mathrm{BiP}$ (spot 8), t-complex protein 1 (spot 36), hsp90binding cochaperone protein (spot 37), UDP-glucose pyrophosphorylase (spot 6), and UDP-galactopyranose mutase (spot 15). In contrast, some genes exhibited greater differences in transcript levels than in protein abundance, such as pyruvate decarboxylase (spot 11), retinol dehydrogenase 8 protein (spot 57), phosphoenolpyruvate carboxykinase (spot 33), ThiJ/PfpI family protein (spot 28), and CDP-alcohol phosphatidyltransferase (spot 35). Some genes exhibited a completely opposite difference in gene expression than the differences observed in protein abundance, including transaldolase (spot 53 and 63), elongation factor 1-gamma (spot 73), 6-phosphogluconate dehydrogenase (spot 55 and 66), elongation factor 2 (spot 47), and kelch domaincontaining protein (spot 71). These contrasting differences in gene expression versus protein abundance may be attributable to posttranslational modification. To confirm the changes in protein abundance observed in proteomics analysis, two proteins (transaldolase and 6-phosphogluconate dehydrogenase) were chosen for Western blot analysis. Green fluorescent protein (GFP)-tagged strains of transaldalose (BcTAL) or 6-phosphogluonate dehydrogenase (BcPGD) in WT or $\Delta b c n o x R$ mutant were constructed by homologous recombination (Fig. 5A). Western blot analysis using GFP antibody indicated that the abundance of both proteins was reduced markedly (Fig. 5B). The results were consistent with those observed in the proteomic analysis.

A

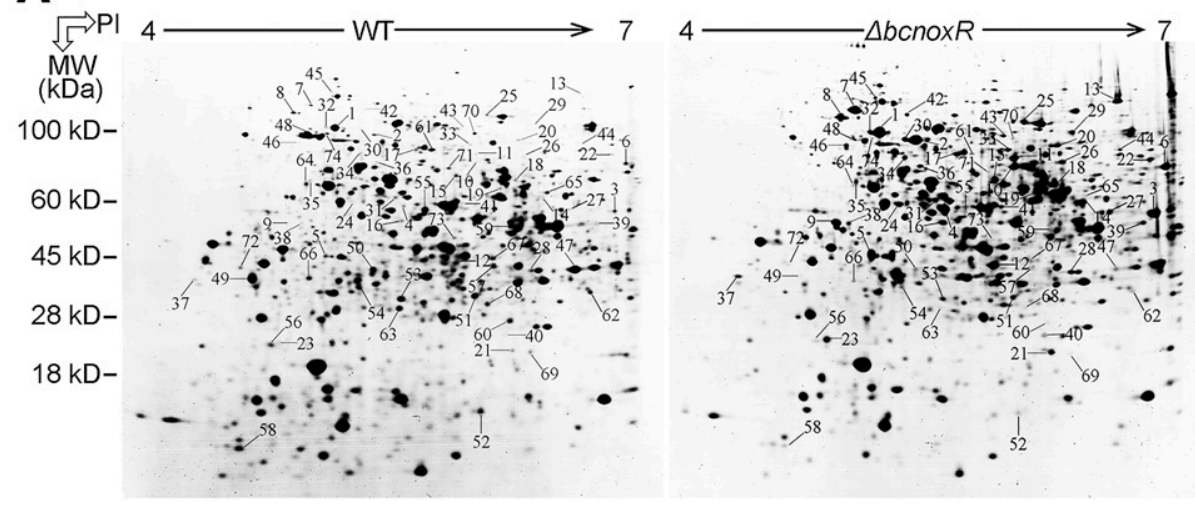

B

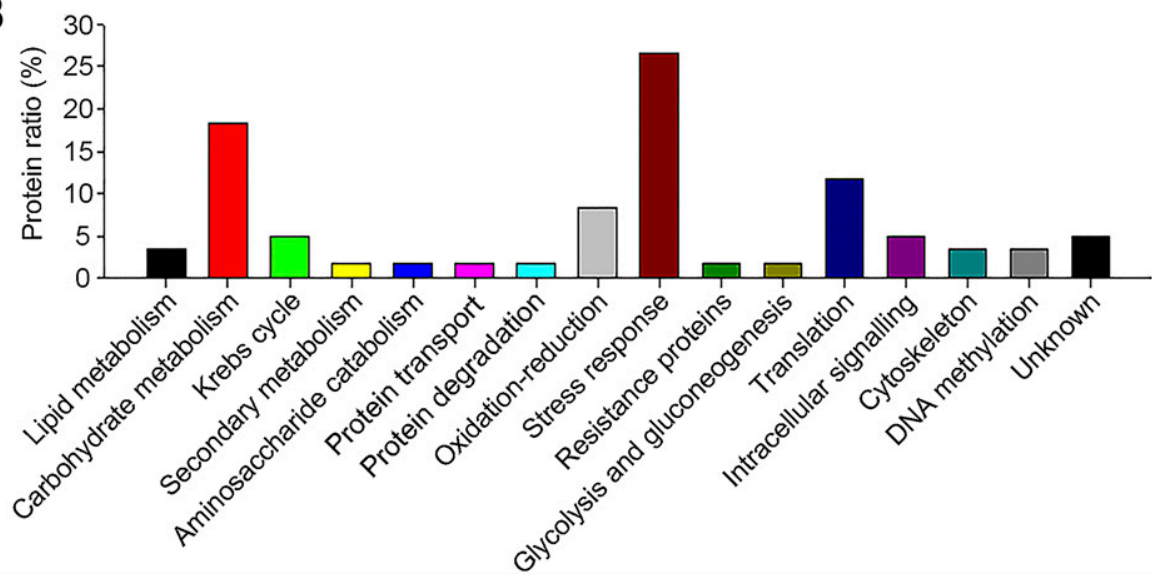

Fig. 2. Comparative proteomic analysis of the wild type (WT) and $\Delta b c n o x R$ mutant reveal potential downstream targets of BcNoxR. A, Images of twodimensional gels illustrating protein spots obtained from wild type (WT) and the $\Delta b c n o x R$ mutant. Proteins (500 $\mu \mathrm{g}$ ) were separated on Immobiline Dry strips with a linear $\mathrm{pH}$ gradient from 4 to 7 in the first dimension and by sodium dodecyl sulfate-polyacrylamide gel electrophoresis in the second dimension. Numbers indicate proteins that were differentially expressed in the $\Delta b c n o x R$ mutant and were, subsequently, identified by mass spectrometry. B, Functional classification of the differentially expressed proteins identified in the proteomic analysis. 
Functional analysis

of BcTAL, BcPGD, BcVWF, and BcKDC.

Four proteins whose abundance was significantly reduced in the $\Delta b c n o x R$ mutant were selected for functional analysis. These proteins included a transaldolase (BcTAL; spots 53 and 63) 6-phosphogluconate dehydrogenase (BcPGD; spots 55 and 66), Von Willebrand factor (VWF) type A domain-containing protein (BcVWF; spots 64 and 72), and a kelch domain-containing

A eukaryotic translation initiation factor 3 subunit $\mathrm{f}$ protein (Bcin04g05810) actin (Bcin16g02020) fimbrin (Bcin04g01050)

UDP-galactopyranose mutase (Bcin05g00580 activator of hsp90 aha1 (Bcin08g06700) phosphoenolpyruvate carboxykinase (Bcin16g00630) cobalamin-independent methionine synthase (Bcin04g06570) DUF1688 domain-containing proteintransferase (Bcin02g01020) isocitrate dehydrogenase subunit 2 (Bcin07g04280) translation elongation factor EF-Tu (Bcin03g07240) ATP citrate lyase subunit (Bcin13g02430) predicted protein (Bcin05g01260) ATP-specific succinyl-CoA synthetase beta subunit (Bcin15g01380) heat shock protein 84 (Bcin10g00300) arm repeat-containing protein (Bcin08g05770) hsp70 (Bcin05g00180) t-complex protein 1 (Bcin16g01640) dnaK-type molecular chaperone BiP (Bcin13g00960) S-adenosylmethionine synthetase (Bcin11g03520) lysyl-tRNA synthetase (Bcin16g00430) pyruvate decarboxylase (Bcin02g06580) 40S ribosomal protein SO (Bcin05g05820) delta-1-pyrroline-5-carboxylate dehydrogenase (Bcin11g06270) thiazole biosynthetic enzyme (Bcin07g06010) UDP-N-acetylglucosamine pyrophosphorylase (Bcin05g00420) inosine 5-monophosphate dehydrogenase (Bcin05g08280) pyruvate decarboxylase (Bcin02g06580)

UTP-glucose-1-phosphate uridylyltransferase (Bcin01g03640) retinol dehydrogenase 8 protein (Bcin06g04940) peroxiredoxin PRX1 (Bcin01g08520)

Von Willebrand factor type A domain (Bcin16g03080)

UDP-N-acetylglucosaminepyrophosphorylase (Bcin05g00420)

proteasome subunit alpha type 6 (Bcin09g03160) thiazole biosynthetic enzyme (Bcin07g06010)

translation initiation factor elF3 (Bcin15g04490)

kelch domain-containing protein (Bcin09g02320)

6-phosphogluconate dehydrogenase (Bcin07g02610) heat shock $70 \mathrm{kDa}$ protein 2 (Bcin03g06600) hsp70 (Bcin05g00180)

6-phosphogluconate dehydrogenase (Bcin07g02610) elongation factor 2 (Bcin07g01540) conidial pigment biosynthesis scytalone dehydratase Arp 1 (Bcin03g08110) catalase protein (Bcin09g04400 elongation factor 1-gamma (Bcin03g05960) transaldolase (Bcin03g03360) retinol dehydrogenase (Bcin12g06430) Von Willebrand factor type A domain (Bcin16g03080) cyanovirin-N family protein (Bcin01g10500) transaldolase (Bcin03g03360) heat shock protein 84 (Bcin10g00300)
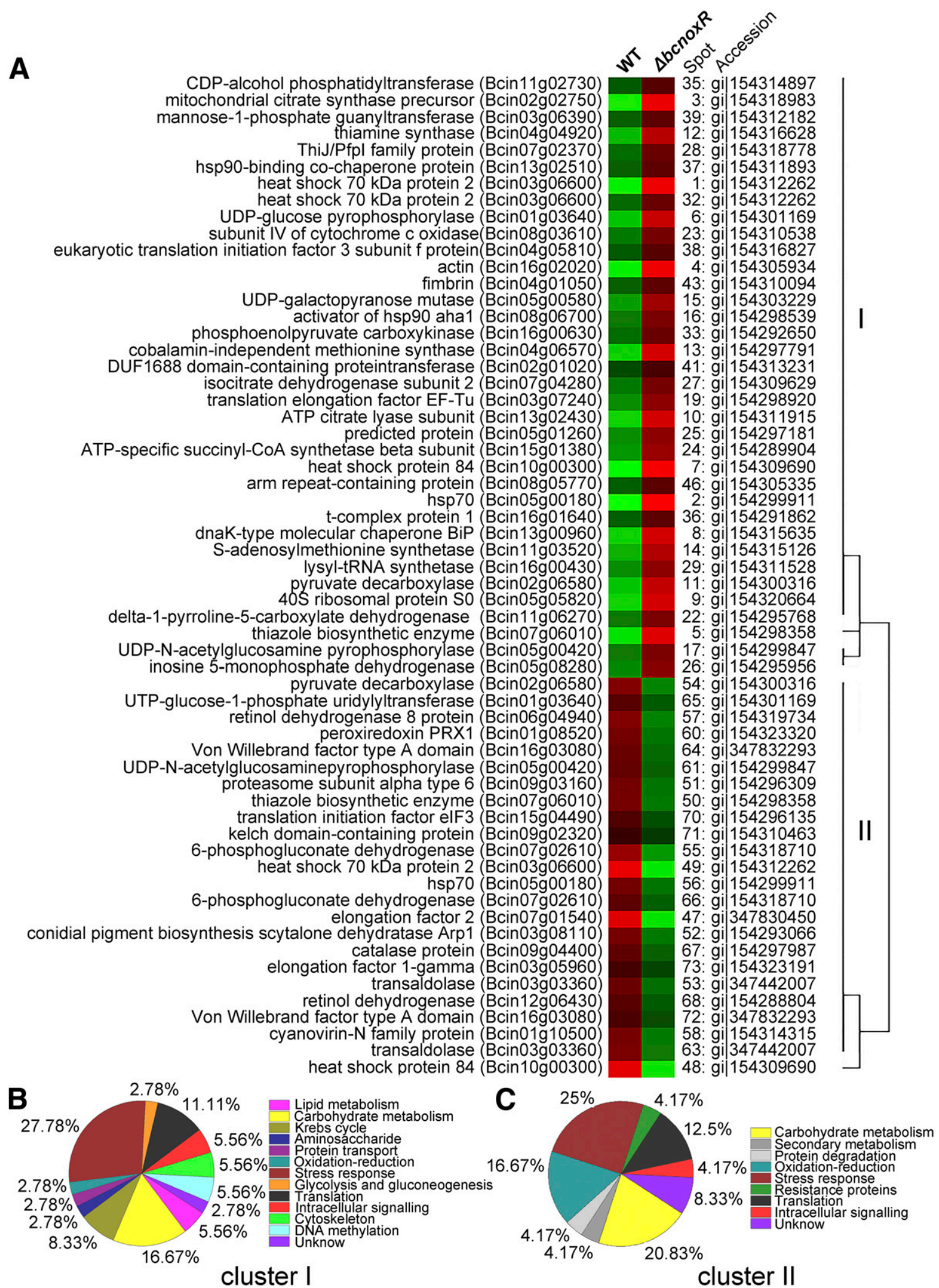

Fig. 3. Hierarchical clustering of differences in protein abundance in the wild type (WT) and $\Delta b c n o x R$ mutant. A, Spots whose abundance differed significantly between the WT and $\Delta b c n o x R$ mutant in the two-dimensional gels were clustered into cluster I, proteins with significantly higher abundance in the $\Delta b c n o x R$ mutant, and cluster II, proteins with significantly lower abundance in the $\Delta b c n o x R$ mutant. Clustering was based on the percentage of volume of each spot, using the Pearson clustering algorithm. Each row in the heat map indicates a single protein, and each column represents proteins from either the wild type (WT) or the $\Delta b c n o x R$ mutant. The spot and accession numbers and the functional annotation are provided for each protein. $\mathbf{B}$ and $\mathbf{C}$, Functional classification of the proteins in each cluster. 
protein (BcKDC; spot 71). They are important for the function of NOX or have overlap functions with NOX. Transaldolase is an enzyme in the pentose phosphate pathway that generates reduced nicotinamide adenine dinucleotide phosphate (NADPH) for reductive biosynthesis. The enzyme, 6-phosphogluconate dehydrogenase is also in the pentose phosphate pathway. It catalyzes the oxidative decarboxylation of 6-phosphogluconate into ribulose 5-phosphate, with a concomitant reduction of NADP to NADPH. The VWF is a large, multimeric glycoprotein involved in hemostasis. Proteins bearing VWF domains play a role in numerous biological processes, including cell adhesion, migration, homing, pattern formation, and signal transduction and they also have the ability to interact with a large array of ligands. The biosynthesis of VWF requires the formation of disulfide bonds in the acidic environment of the Golgi apparatus (Sadler 1998). Cysteines are involved in the formation of intrachain disulfide bonds in VWF proteins and mutations that affect cysteine residues cause von Willebrand's disease, which is characterized by reduced VWF storage and secretion (Wang et al. 2012). Kelch domain-containing proteins are a widespread group of proteins that contain multiple Kelch motifs. The Kelch domain-containing proteins are involved in diverse cellular functions, including oxidation, gene expression, and cell organization (Adams et al. 2000).

Knockout mutants of these four genes were separately constructed by homologous recombination (Fig. 6A). The homozygous

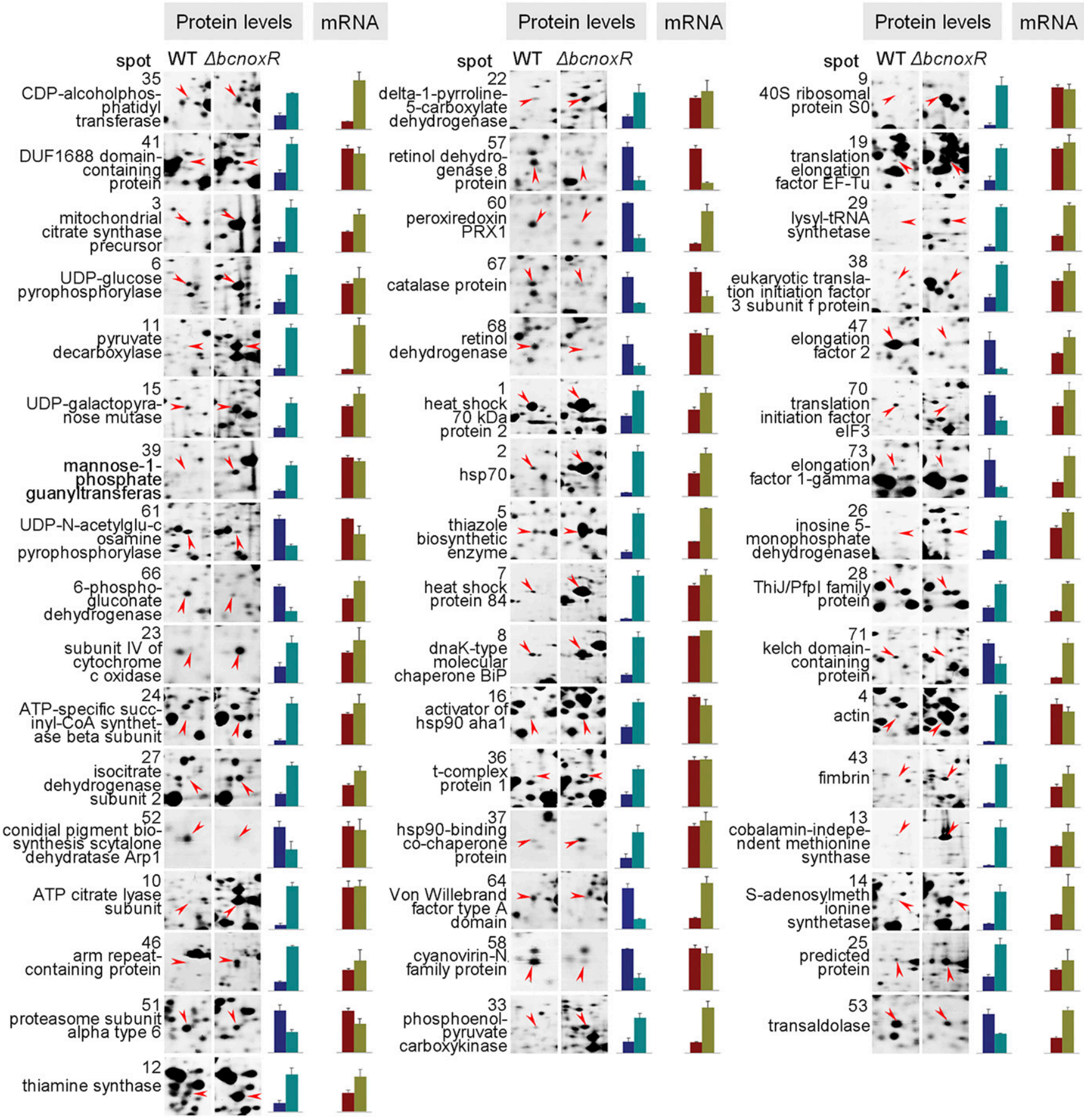

Fig. 4. Comparison of gene transcript profiles and protein abundance values, showing representative images from two-dimensional gels of proteins in the wild type (WT) and the $\Delta$ bcnoxR mutant. The spot number, the functional annotation, and abundance for each protein are provided. Transcript levels were determined by revesr transcription-quantitative polymerase chain reaction. Relative gene expression values are presented after normalization against the tubulin gene, followed by normalization against WT. Gene expression values represent the mean \pm standard deviation of three biological replicates. 
strains of $\Delta b c p g d$ were confirmed by Southern blot analysis (Supplementary Fig. S4). The growth rate of $\Delta b c t a l, \Delta b c v w f$, and $\Delta b c k d c$ was similar to the WT when the strains were cultured on complete medium (CM). In contrast, however, $\Delta b c p g d$ exhibited a reduced rate of growth (Fig. 6B). The sporulation in $\Delta$ bctal and $\Delta b c v w f$ were similar to the WT, while sporulation in $\Delta b c p g d$ and $\Delta b c k d c$ were significantly reduced (Fig. 6C).

BcPGD is involved in the pathogenicity of $B$. cinerea.

Spore suspensions of the four deletion mutants ( $\Delta$ bctal, $\Delta b c p g d, \Delta b c v w f$, and $\Delta b c k d c$ ) were inoculated in wounded apple, tomato, and strawberry fruit and detached tomato leaves, in order to determine their impact on virulence. Mutants of $\Delta b c t a l, \Delta b c v w f$, and $\Delta b c k d c$ did not cause a significant reduction in lesion diameter, relative to the WT, on apple fruit or on detached tomato leaves (Supplementary Fig. S5). The mutant strains $\Delta b c p g d 1, \Delta b c p g d 2$, and $\Delta b c p g d 3$, however, exhibited an obvious reduction in virulence, as measured by lesion size, on apple, strawberry, and tomato fruits (Fig. 7). In contrast, the complementation mutants of bcpgd exhibited the same level of virulence as the WT on inoculated fruits (Fig. 8). On detached tomato leaves, however, the virulence of the $\Delta b c p g d$ mutants was the same as the WT (Fig. 7). These results suggest that BcPGD, whose abundance is affected by NOX, may function in the pathogenesis of $B$. cinerea on various fruits.

\section{DISCUSSION}

ROS are both detrimental and indispensable to the survival of aerobic organisms (Egan et al. 2007). Oxidative phosphorylation

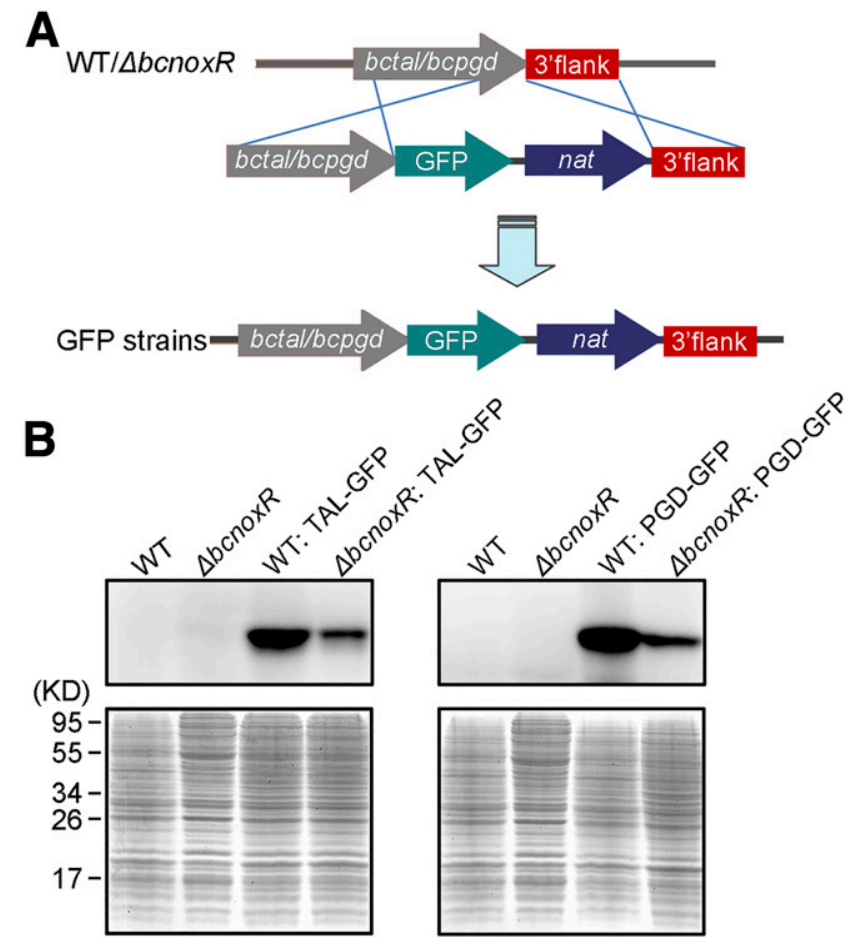

Fig. 5. Western blot analysis of the expression of BcTAL and BcPGD in wild type (WT) and $\Delta b c n o x R$. A, Strategy for constructing gren fluorescent protein (GFP)fusion strains of BcTAL and BcPGD. Each gene without the stop codon, $g f p$ gene, nourseothricin-resistance gene (nat) region, and about 1,000 bp immediately downstream of the gene bctal/bcpgd were separately amplified and ligated by fusion polymerase chain reaction. GFP-fusion strains were generated by replacing the stop codon of bctal/bcpgd with GFP and a nourseothricin-selectable marker through transformation of protoplasts of WT or $\Delta b c n o x R$ strain. B, Western blot analysis as performed to show the changes in protein abundance of BcTAL and $\mathrm{BcPGD}$ in the $\Delta b c n o x R$ mutant. The proteins of WT or the $\Delta b c n o x R$ strain were used as control. Coomassie brilliant blue staining was used as a loading control. is a fundamental of energy metabolism in all aerobic organisms. In the process of oxidative phosphorylation, the four-electron $\mathrm{O}_{2}$ is reduced to $\mathrm{H}_{2} \mathrm{O}$, providing oxygen molecules for the formation of ROS (partially reduced reactive metabolites of $\left.\mathrm{O}_{2}\right)($ Muller et al. 2004). Considering the potential of ROS to damage proteins, DNA, and lipids, ROS were once regarded as inevitable toxic by-products of metabolism. Growing evidence now indicates, however, that ROS are not only pernicious by-products but also indispensable participants in cell signaling and regulation (Aguirre et al. 2005; Tian et al. 2013). Egan et al. (2007) reported that ROS play a crucial role in the growth and virulence of the fungal pathogen Magnaporthe grisea.

Although ROS play multiple roles in cellular processes, its function in pathogenicity remains to be investigated. In the present study, the regulatory subunit of NOX (BcNoxR) was found to be required for optimum growth and virulence of the fungal pathogen $B$. cinerea. These results are consistent with the study by Segmüller et al. (2008), who reported that a $\Delta b c n o x R$ mutant exhibits a reduced growth rate on CM plates, cannot form sclerotia, and has a severe reduction in virulence of $B$. cinerea. We conducted a comparative proteomic analysis comparing the protein abundance in WT $B$. cinerea with that in a $\Delta b c n o x R$ mutant, in order to investigate the molecular mechanisms by which BcNoxR functions in growth and virulence.

Proteomics have been widely used as a method for studying specific proteins that play a role in the virulence of plant pathogens or in their response to abiotic stresses (GonzálezRodríguez et al. 2015; Qin et al. 2007). Although highthroughput next-generation sequencing is efficient for studying global changes in gene transcript levels, it cannot be used to predict concomitant levels of protein abundances, since discrepancies often exist between expression and abundance levels, for a variety of reasons. Protein abundance can be determined by many regulatory mechanisms other than transcription, including mRNA localization, translational regulation, and protein turnover (Pradet-Balade et al. 2001). The function of proteins can also be altered through posttranslational modifications or targeted proteolysis (Pradet-Balade et al. 2001). Therefore, we utilized a proteomic approach to explore the differences in protein abundance between the $\Delta b c n o x R$ mutant and WT strains of B. cinerea.

Several proteins related to oxidation reduction were identified in our analysis, including delta-1-pyrroline-5-carboxylate dehydrogenase, peroxiredoxin, catalase, and retinol dehydrogenase. Among these proteins, delta-1-pyrroline-5-carboxylate dehydrogenase is involved in proline metabolism. Proline accumulation in Saccharomyces cerevisiae cells make it more tolerant to freezing, desiccation, or oxidative stress (Matsuura and Takagi 2005; Morita et al. 2002, 2003; Terao et al. 2003). Proline has also been reported to be a singlet oxygen quencher (Matysik et al. 2002). Proline treatment can prevent fungi and yeast from programmed cell death by diminishing ROS levels (Chen and Dickman 2005). Peroxiredoxin has been demonstrated to act not only as an antioxidant but also to regulate hydrogen peroxide-mediated signal transduction (Rhee et al. 2005). Catalase was reported to play an essential role in p53-mediated ROS regulation (Kang et al. 2013). The p53/p53R2-catalase and p53/PIG3-catalase pathways were shown to be critically involved in intracellular ROS regulation under physiological conditions and during the response to DNA damage (Kang et al. 2013). Catalase was also suggested to be the molecular linker between ROS and PCD signaling in Arabidopsis (Hackenberg et al. 2013). Retinol dehydrogenase has been reported to be a mitochondrial short-chain dehydrogenase/reductase with retinaldehyde reductase activity (Belyaeva et al. 2008). The detection of changes in the abundance of these proteins in the $\Delta$ bcnoxR mutant indicated that NOX may affect the level of ROS through influencing the expression of these proteins. Two 
methylation proteins, cobalamin-independent methionine synthase and $S$-adenosylmethionine synthetase, were also identified in our analysis. Cobalamin-independent methionine synthase is a methyl transferase and catalyzes the synthesis of the amino acid methionine by transferring a methyl group from methyl tetrahydrofolate to homocysteine. Oxidative stress was reported to inactivate cobalamin-independent methionine synthase in Escherichia coli (Hondorp and Matthews 2004). In our study, this protein was upregulated in the $\Delta b c n o x R$ mutant, perhaps due to the low level of ROS produced by NOX. $S$-adenosylmethionine synthetase is an enzyme that creates the methyl donor $S$-adenosylmethionine (SAM) by facilitating a reaction between methionine and ATP. SAM facilitates DNA methylation, which then blocks gene transcription. SAM is also involved in gene transcription, cell proliferation, and the production of secondary metabolites. In Aspergillus nidulans, overexpression of $S$-adenosylmethionine synthetase resulted in aberrant growth (Gerke et al. 2012). Abnormal growth was also observed in our $\Delta b c n o x R$ mutants, in which $S$-adenosylmethionine synthetase is up-regulated, relative to expression in the WT strain of $B$. cinerea. It seems plausible

A

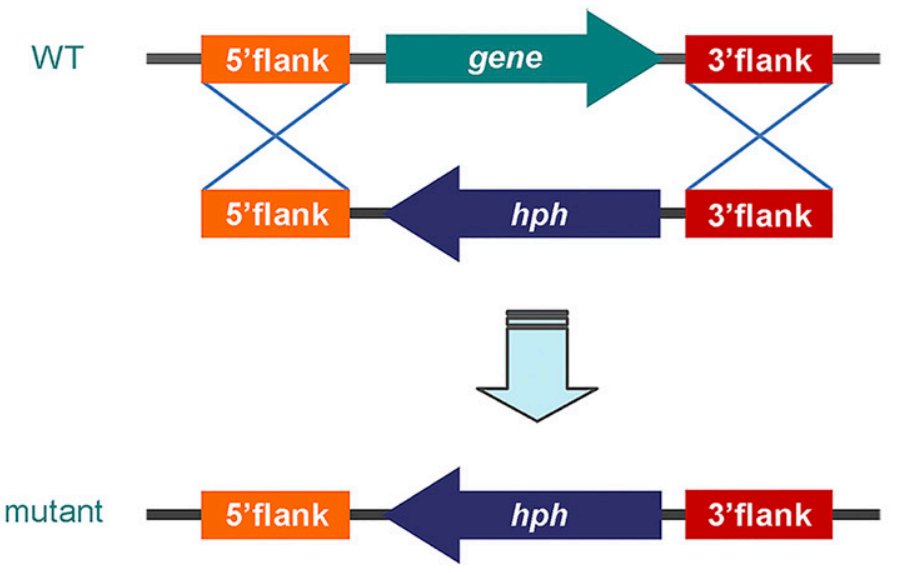

B

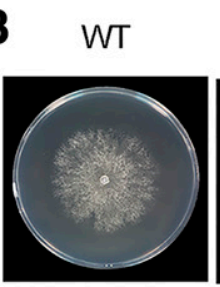

$\Delta b c t a l$

$\Delta b c p g d$
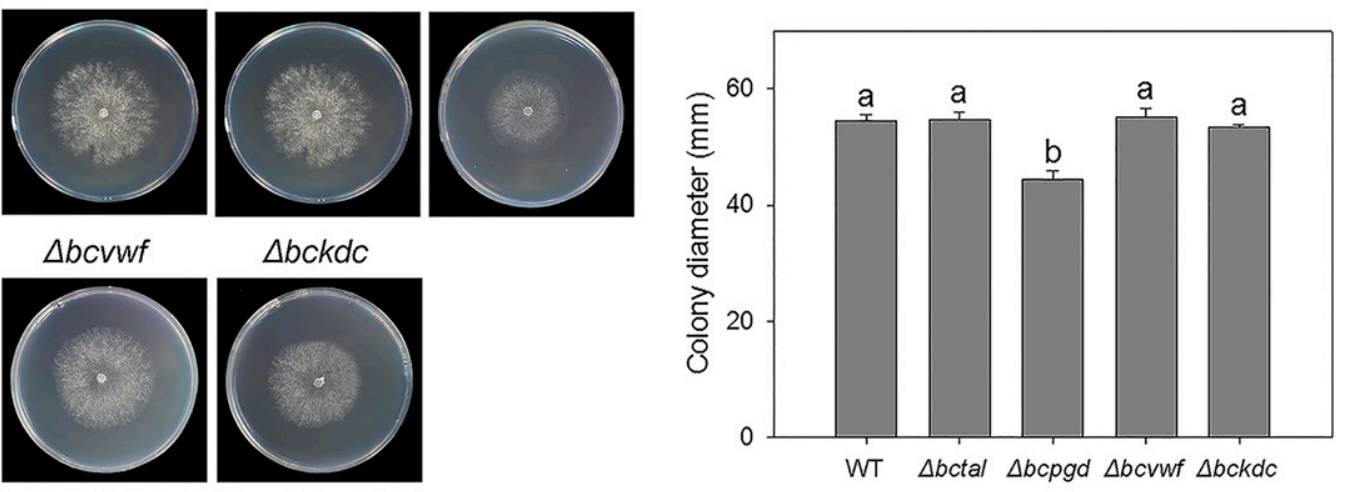

C WT

$\Delta b c t a l$
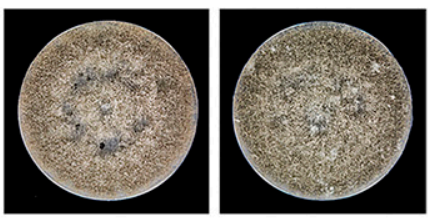

$\Delta b c p g d$

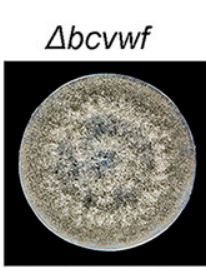

$\Delta b c k d c$
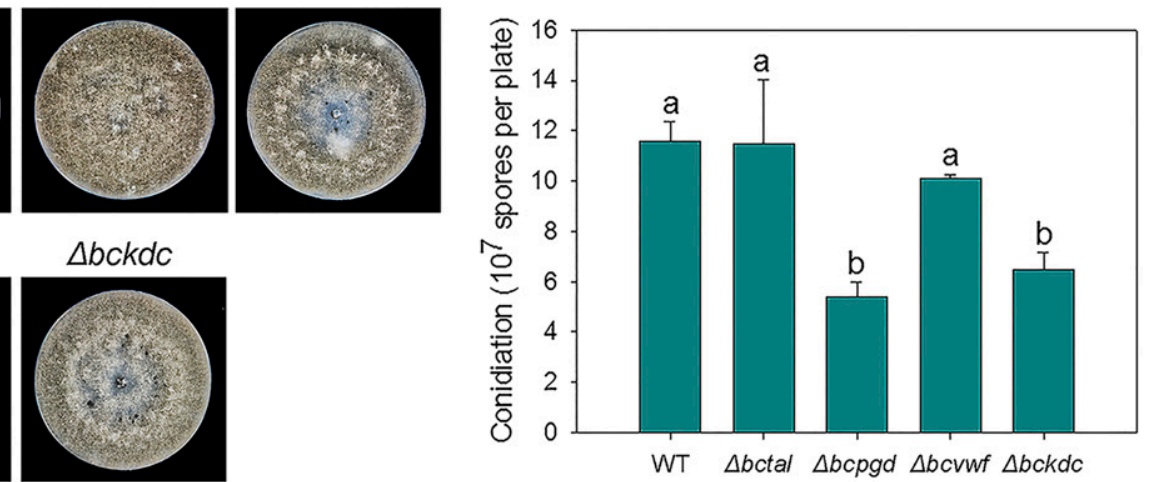

Fig. 6. Phenotypic analysis of deletion mutants constructed using genes whose respective protein abundance was significantly lower in the $\Delta b c n o x R$ mutant than in the wild type (WT). A, Replacement strategy for constructing deletion mutants of differentially expressed genes. Flank L and Flank R were amplified using primer pairs appropriate for each specific gene. Deletion mutants were generated by replacing the open reading frame with a hygromycin B-selectable marker through transformation of protoplasts of the WT strain. B, Representative photographs of fungal growth and colony diameters of WT, $\Delta b c t a l, \Delta b c p g d$, $\Delta b c v w f$, and $\Delta b c k d c$ mutant strains after $48 \mathrm{~h}$ of growth on complete medium (CM). C, Representative photographs of sporulating cultures and levels of conidiation of the WT, $\Delta b c t a l, \Delta b c p g d, \Delta b c v w f$, and $\Delta b c k d c$ mutant strains after $288 \mathrm{~h}$ of growth on $\mathrm{CM}$. The strains were cultured at $22^{\circ} \mathrm{C}$. Data presented are the mean \pm standard deviation $(n=3)$. Columns with different letters are significantly different from each other, using the least significant difference test $(P<0.05)$. 
that BcNoxR may affect growth by impacting the protein abundance of $S$-adenosylmethionine synthetase.

Sixteen proteins related to stress response were identified in the present study, including heat-shock proteins, chaperones, and proteins involved in thiamine (vitamin B1) synthesis. Heatshock protein 90 has been reported to regulate NOX activity and is necessary for superoxide production (Chen et al. 2011). The expression and activity of molecular chaperones have been
A

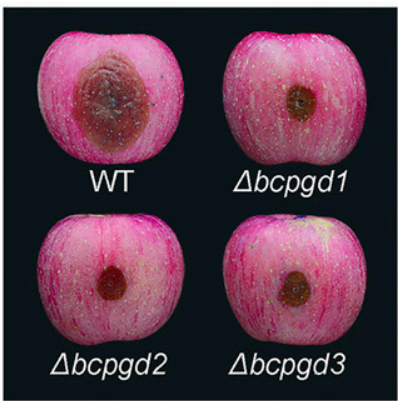

C

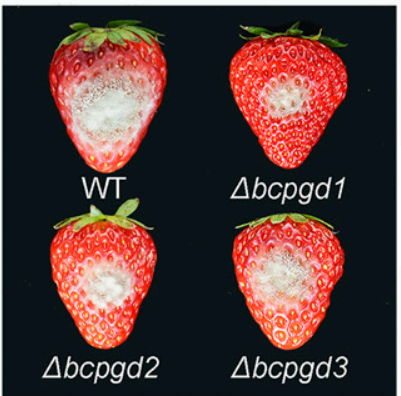

E

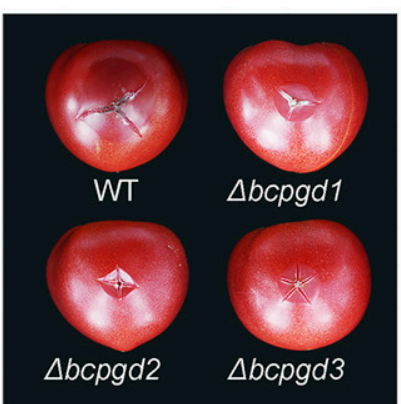

G

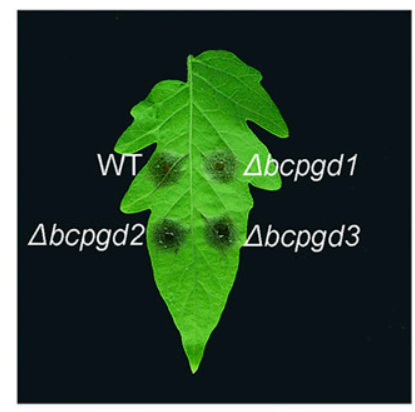

B

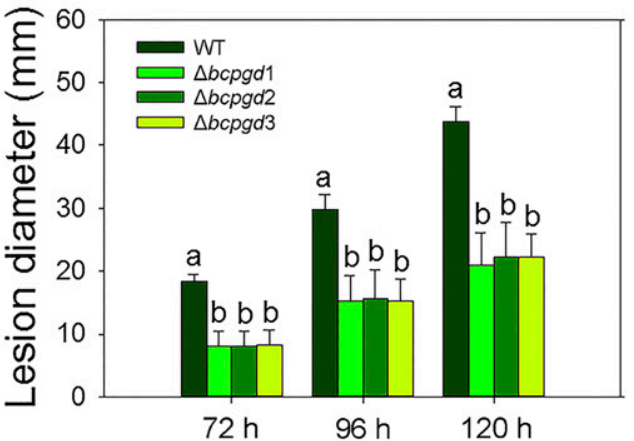

D

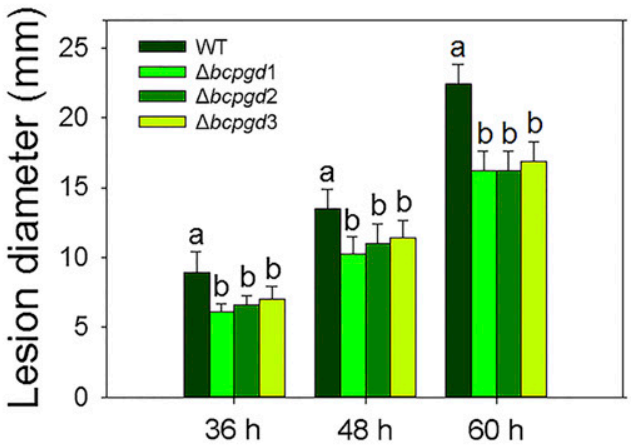

F

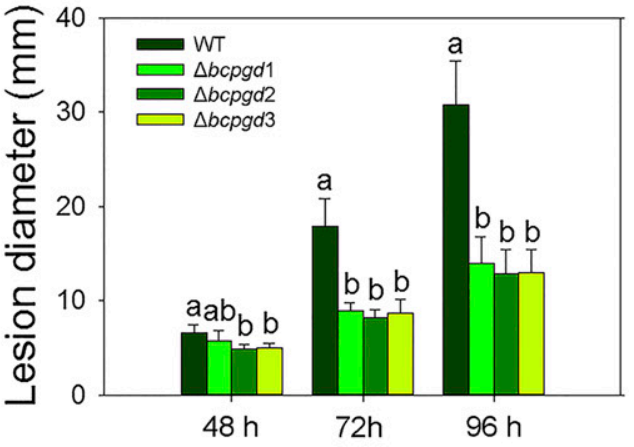

H

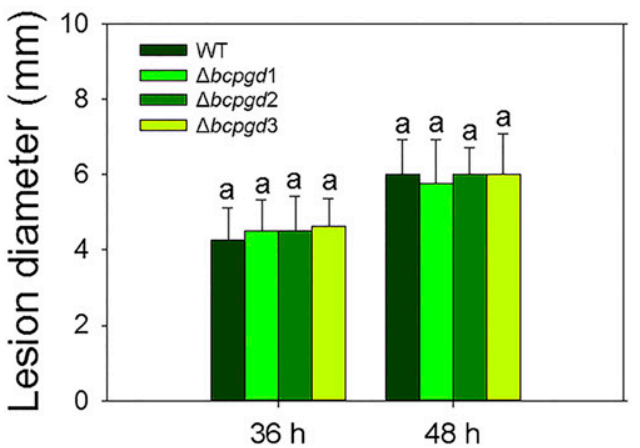

Fig. 7. BcPGD is involved in the pathogenicity of Botrytis cinerea. A and B, Representative photograph (taken at $120 \mathrm{~h}$ postinoculation [hpi]) of disease symptoms and measurements of lesion diameters, respectively, in apples inoculated with wilt type (WT), $\Delta b c p g d 1, \Delta b c p g d 2$, and $\Delta b c p g d 3$ strains of Botrytis cinerea. C and D, Representative photograph (taken at 60 hpi) of disease symptoms and measurements of lesion diameters, respectively, in strawberries inoculated with WT, $\Delta b c p g d 1, \Delta b c p g d 2$, and $\Delta b c p g d 3$ strains of $B$. cinerea. $\mathbf{E}$ and $\mathbf{F}$, Representative photograph (taken at 96 hpi) of disease symptoms and measurements of lesion diameters, respectively, in tomatoes inoculated with WT, $\Delta$ bcpgd1, $\Delta$ bcpgd2, and $\Delta b c p g d 3$ strains of $B$. cinerea. $\mathbf{G}$ and $\mathbf{H}$, Representative photograph (taken at 48 hpi) of disease symptoms and measurements of lesion diameters, respectively, in tomato leaves inoculated with WT, $\Delta$ bcpgd1, $\Delta$ bcpgd2, and $\Delta b c p g d 3$ strains of B. cinerea. Columns with different letters at the same time point are significantly different from each other, using the least significant difference test $(P<0.05)$. 
shown to be tightly regulated during oxidative stress at both the transcriptional and posttranslational level (Niforou et al. 2014). Thiazole biosynthetic enzyme and thiamine synthase are involved in vitamin B1 synthesis. Thiamine diphosphate, the active form of vitamin B1, is a universal cofactor involved in pivotal cellular pathways, which could be modulated under stress conditions to ensure cell survival. Vitamin B1 has been reported to play a protective role in the response of plants to abiotic stress and yeast against oxidative stress (Ahn et al. 2005; Kowalska et al. 2012). The identification of several stress response proteins in the $\Delta b c n o x R$ mutant suggests that $\mathrm{BcNoxR}$ is also involved in the stress response in $B$. cinerea.

NOX utilizes NADPH and oxygen as a substrate to generate superoxide. In this study, we identified two proteins, BcTAL
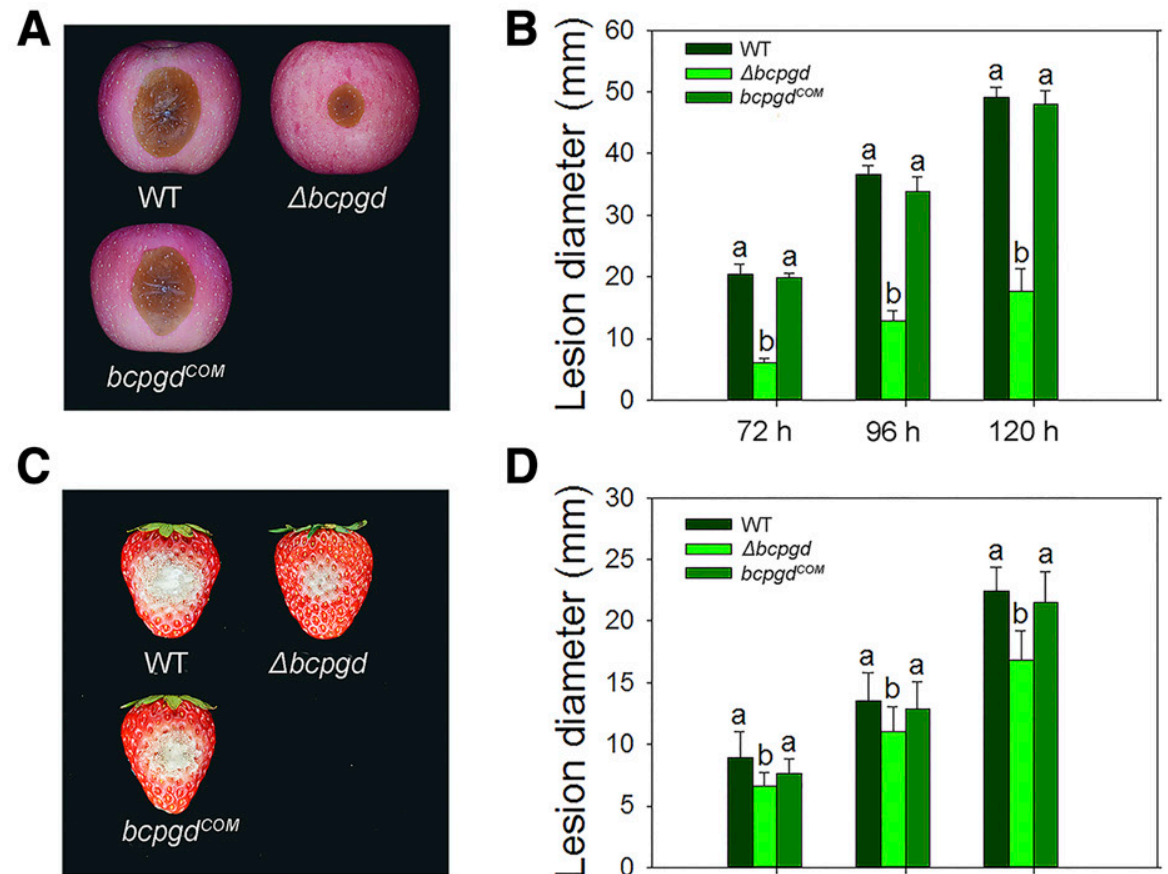

D

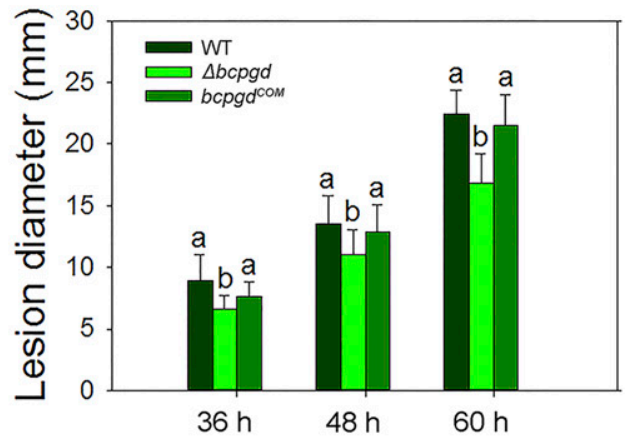

E

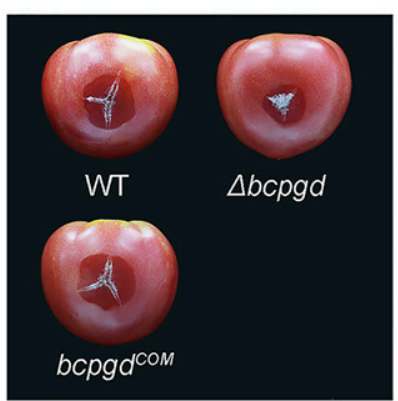

G

$\mathbf{F}$
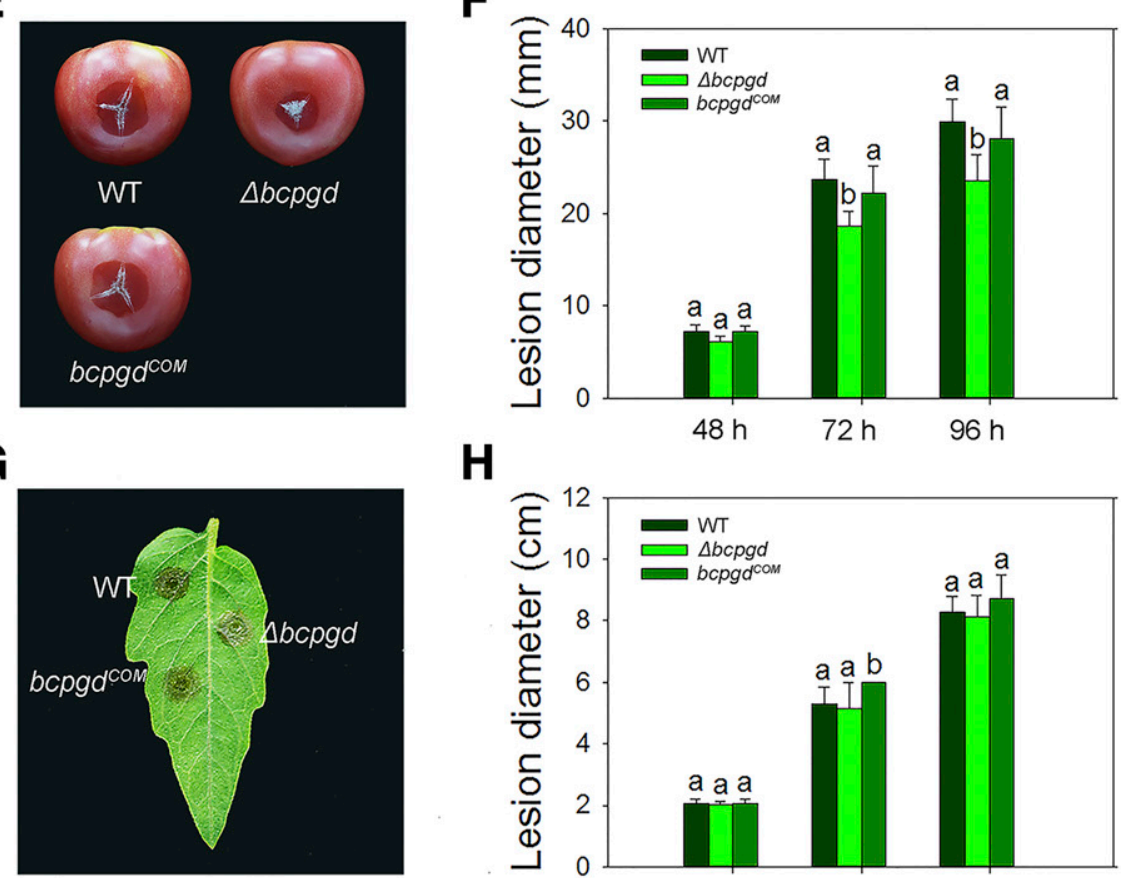

H

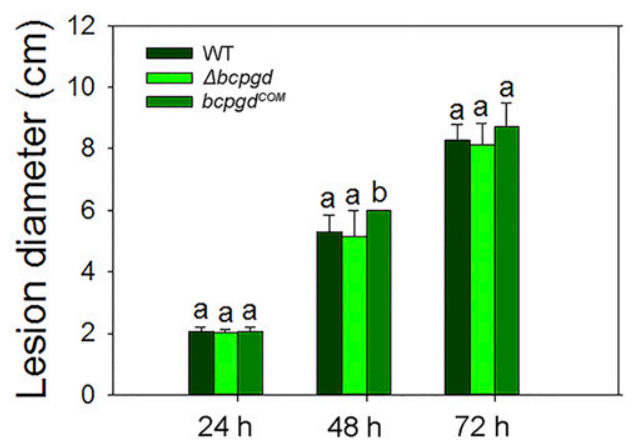

Fig. 8. Complementation of bcpgd in the $\Delta b c p g d$ mutant restores maximum virulence. A and $\mathbf{B}$, Representative photograph (taken at $120 \mathrm{~h}$ postinoculation [hpi]) of disease symptoms and measurements of lesion diameters, respectively, in apple fruit inoculated with wild type (WT), $\Delta b c p g d$, and $b c p g d d^{C O M}$ strains of Botrytis cinerea. C and D, Representative photograph (taken at $60 \mathrm{hpi}$ ) of disease symptoms and measurements of lesion diameters, respectively, in strawberry fruit inoculated with WT, $\Delta b c p g d$, and $b c p g d^{C O M}$ strains of B. cinerea. $\mathbf{E}$ and $\mathbf{F}$, Representative photograph (taken at 96 hpi) of disease symptoms and measurements of lesion diameters, respectively, in tomato fruit inoculated with WT, $\Delta$ bcpgd, and bcpgd $^{C O M}$ strains of B. cinerea. $\mathbf{G}$ and $\mathbf{H}$, Representative photograph (taken at $48 \mathrm{hpi}$ ) of disease symptoms and measurements of lesion diameters, respectively, in tomato leaves inoculated with WT, $\Delta b c p g d$, and bcpgd $d^{\text {CoM }}$ strains of $B$. cinerea. Columns with different letters at the same time point are significantly different from each other, using the least significant difference test $(P<0.05)$. 
and BcPGD, that are both involved in NADPH generation. Both proteins exhibited lower abundance in the $\Delta b c n o x R$ mutant (Figs. 2-4) than in the WT. TAL, which links the pentose phosphate pathway to glycolysis, is a key enzyme that is responsible for the generation of NADPH (Banki et al. 1996). Dysfunction of TAL in mammals results in liver cirrhosis and hepatosplenomegaly (Verhoeven et al. 2001). TAL is also a potent autoimmune target in patients with multiple sclerosis (Colombo et al. 1997). PGD catalyzes the oxidative decarboxylation of 6-phosphogluconate to ribulose 5-phosphate and $\mathrm{CO}_{2}$, with a concomitant reduction of NADP to NADPH (Adams et al. 1994). PGD is generally assumed to be at the nexus of many essential metabolic pathways. Dysfunction of the various pathways that PGD participates in has been shown to be the basis of many human diseases (Stanton 2012). We constructed mutant strains in which either gene was deleted, in order to investigate the function of BcTAL and BcPGD. Phenotypic observations indicated that the virulence of $B$. cinerea on apple, tomato, and strawberry fruits was reduced in $\Delta b c p g d$ but not $\Delta b c t a l$. Nox2-NoxR significantly affects the virulence of Magnaporthe oryzae on rice through appressorium formation (Ryder et al. 2013). To further investigate the mechanisms by which BcPGD affects virulence, the impact of $\Delta b c p g d$ on appresorium formation and infection was investigated. With this assay, we observed that, although BcPGD affects the virulence of $B$. cinerea, it has no effect on the formation of appressoria or infection. The virulence of $B$. cinerea can be affected by many factors, such as secretion of extracellular proteins, production of ROS, and generation of toxins (Choquer et al. 2007; Rolke et al. 2004; Siewers et al. 2005; ValetteCollet et al. 2003). Sporulation and growth rate of $\Delta b c p g d$ were reduced compared with that of wild type. The reduction of growth rate may be one of the factors that affect virulence. Collectively, these findings indicate that BcPGD may affect the virulence of $B$. cinerea through other pathways. We have assayed an interaction between BcPGD and BcNoxR using a yeast two-hybrid system (Supplementary Fig. S6), however, no obvious interactions were observed between these two proteins in the current study. This suggests that BcNoxR may affect BcPGD indirectly.

\section{MATERIALS AND METHODS}

\section{Fungal strains and culture conditions.}

B. cinerea (B05.10) was employed as the WT control and served as the recipient strain for homologous recombination to generate gene deletion mutants. $B$. cinerea cultures were normally maintained at $22^{\circ} \mathrm{C}$ on potato dextrose agar (PDA) or a CM.

\section{Proteins isolation and 2D gel electrophoresis.}

Conidia $\left(1 \times 10^{5}\right.$ spores per milliliter $)$ of the WT or a specific mutant were cultured in PDB liquid medium at $180 \mathrm{rpm}$ for $48 \mathrm{~h}$. Mycelia were collected and washed thoroughly with $\mathrm{KC}$ buffer $(0.6 \mathrm{M} \mathrm{KCl}, 50 \mathrm{mM} \mathrm{CaCl} 2)$ and were vigorously ground into a fine powder, using liquid nitrogen. Proportional protein extraction buffer $(0.5 \mathrm{M}$ Tris- $\mathrm{HCl}[\mathrm{pH} 8.3], 2 \%$ [vol/vol] Nonidet P-40, $20 \mathrm{mM} \mathrm{MgCl} 2,2 \%$ [vol/vol] b-mercaptoethanol, and $1 \mathrm{mM}$ phenylmethylsulfonyl fluoride) was added to the powder and the mixture was, then, thoroughly mixed. The homogenate was centrifuged at $25,000 \times g$ for $20 \mathrm{~min}$ to remove mycelial debris. An equal volume of Tris- $\mathrm{HCl}(\mathrm{pH} 7.5)-$ buffered phenol was added to the supernatant, was shaken for $10 \mathrm{~min}$, and was centrifuged at $20,000 \times g$ for $15 \mathrm{~min}$, to retain the proteins within the phenol phase. Five volumes of saturated ammonium acetate in methanol were used to precipitate the proteins at $-20^{\circ} \mathrm{C}$ overnight. The proteins were pelleted by centrifugation at $20,000 \times g$ for $30 \mathrm{~min}$ and were washed twice with ice-cold saturated ammonium acetate in methanol, followed by two rinses with ice-cold acetone.

The extracted proteins from the WT and specific mutant strains were solubilized in isoelectric focusing (IEF) buffer (7 M urea, $2 \mathrm{M}$ thiourea, 4\% CHAPS, $1 \%$ dithiothreitol, and $2 \%$ carrier ampholytes), were quantified using the Bradford assay, and were loaded onto $13-\mathrm{cm}, \mathrm{pI} 4$ to 7 linear immobilized $\mathrm{pH}$ gradient (IPG) strips ( $500 \mu \mathrm{g}$ protein per gel) by rehydration for $15 \mathrm{~h}$. The first-dimensional IEF was carried out at $20^{\circ} \mathrm{C}$ for a total of $20 \mathrm{kVh}$ on an Ettan IPGphor unit following the user's manual (GE Healthcare Bio-Sciences AB). IPG strips were equilibrated according to the method described by Qin et al. (2011). After equilibration, the strips were transferred to $15 \%$ SDS-PAGE gels for 2D electrophoresis, which was performed at a constant $30 \mathrm{~mA}$ per gel. Gels were stained with Coomassie brilliant blue R-250 solution (50\% methanol, $15 \%$ acetic acid, and $0.1 \%$ Coomassie brilliant blue R-250) and were scanned using a flatbed scanner (GE Healthcare Bio-Sciences AB). Image Master 2D Elite software (GE Healthcare Bio-Sciences $\mathrm{AB}$ ) was then employed to compare protein abundance in the 2D gel images. The relative abundance of a protein spot was determined by spot volume, which was normalized as a percentage of the total volume of all spots on the gel. Spots that were present in gels of all three biological replicates and whose abundance levels were significantly different (Student's $t$ test) in the mutant strain compared with the WT were excised for protein identification.

\section{In-gel digestion and nano-liquid chromatography MS/MS.}

For in-gel digestion, protein spots were excised from the gels, were destained with $50 \mathrm{mM} \mathrm{NH}_{4} \mathrm{HCO}_{3}$ in $50 \%$ (vol/vol) methanol, were dried using a vacuum centrifuge, and then, were digested with $10 \mathrm{ng}$ of trypsin per microliter (Promega) for $16 \mathrm{~h}$ at $37^{\circ} \mathrm{C}$. Digested peptides were extracted with $0.1 \%$ trifluoroacetic acid (TFA) in 50\% acetonitrile. The peptides were then lyophilized and resuspended in $5 \mathrm{mg} / \mathrm{ml}$ matrix solution (a-cyano-4-hydroxycinnamic acid in 50\% acetonitrile containing $0.1 \%$ TFA) before spotting onto matrix-assisted laser desorption ionization (MALDI) target plates. Spectra were obtained using a MALDI-TOF/TOF mass spectrometer (4800 Proteomics Analyzer; Applied Biosystems). MS spectra were obtained by employing 1,600 laser shots per spectrum, while MS/MS spectra were acquired with 2,500 laser shots per fragmentation spectrum.

To eliminate trypsin autolytic peptides as well as other background ions, the ten strongest peaks of each MS spectra were selected as precursor ions. Spectral analyses were conducted with 4000 Series Explorer TM software (Applied Biosystems), using a signal to noise threshold of 10 and a minimum area of 100. The generated peak lists were entered into the Mascot MS/MS Ions Search program and were annotated by blasting the obtained sequences against the National Center for Biotechnology Information (NCBI) nonredundant database. Search parameters were set as: database, NCBInr; enzyme, trypsin; max missed cleavages, 1; taxonomy, fungi; fixed modifications, carbamidomethyl $(\mathrm{C})$; variable modifications, oxidation (M); peptide mass tolerance, $0.2 \mathrm{Da}$; fragment mass tolerance, 0.3 Da.

\section{RNA isolation and RT-qPCR.}

Fungal total RNA was isolated from the same mycelia collected for the proteomic analyses. RNA was extracted using TRNzol Reagent (Tiangen Biotech), and first-strand cDNA was synthesized using a PrimeScript RT reagent kit with gDNA eraser (Takara). RT-qPCR analyses were performed using a 
Step One Plus Real-Time PCR system (Applied Biosystems) with SYBR premix ex Taq (Takara). Primer pairs were designed using Primer Express software 3.0 (Applied Biosystems). Primer pairs used in the study are listed in Supplementary Table S2. The PCR conditions were as follows: $95^{\circ} \mathrm{C}$ for $30 \mathrm{~s}, 40$ cycles of $95^{\circ} \mathrm{C}$ for $5 \mathrm{~s}$, and $60^{\circ} \mathrm{C}$ for $30 \mathrm{~s}$ The change in fluorescence of SYBR green in every cycle was monitored by the system software and the threshold cycle $\left(C_{t}\right)$ over the background was calculated. The $B$. cinerea tubulin gene was used as an endogenous control for normalization. Relative expression levels were calculated using the $2^{-\Delta \Delta \mathrm{Ct}}$ method (Livak and Schmittgen 2001).

\section{Generation of deletion mutants and complementation strains.}

Approximately $1 \mathrm{~kb}$ of flanking sequence proximal to the initiation codon and immediately downstream of the termination codon of bcnoxR, bcpgd, bctal, VWF type A domain containing protein (bcvwf), and bckdc were amplified. Primers utilized are listed in Supplementary Table S3. The PCR products of these genes were purified and were ligated into pLOB7 to flank the hph marker in the reverse direction to obtain replacement vectors. The replacement vectors were amplified with high fidelity enzyme and the resulting DNA fragments were directly introduced into protoplasts of B05.10 after purification. Protocols to generate protoplasts and conduct transformation were adopted from those reported in Zhang et al. (2014). Transformants were initially selected on hygromycin and were subsequently confirmed by PCR.

Single conidia were isolated in order to purify the genedisruption mutant candidates. Southern hybridization analysis was performed to confirm single-copy genomic integration. Genomic DNA obtained from the B05.10 WT and three deletion mutants was digested with appropriate restriction enzyme pairs. DNA gel blot analysis was performed with the downstream flanking region of the targeted gene or the P-OliC section as a probe.

For complementation, the complete target gene locus carrying the $5^{\prime}$ region (approximately $2 \mathrm{~kb}$ of upstream nucleotides) and the ORF of a specific gene were obtained from WT genomic DNA and were cloned into the pNAN-OGG vector (Schumacher 2012) containing resistance to nourseothricin. The constructed plasmid was used to transform the corresponding mutant. The transformants were selected on nourseothricin and were confirmed by PCR.

\section{Analysis of phenotypes.}

Radial growth assay. Hyphal disks of the WT and deletion mutants, with diameters of approximately $1 \mathrm{~mm}$, were removed from the growing edges of cultures growing on PDA. The disks were excised using a cut $200-\mu l$ pipette tip having an inner diameter of approximately $1 \mathrm{~mm}$. Cultures were grown at $22^{\circ} \mathrm{C}$ and radial growth measurements were taken at 24,48 , and $72 \mathrm{~h}$ after the transfer of the mycelia disk to new PDA plates.

Sporulation assay. Spores were harvested at $288 \mathrm{~h}$ after inoculation by flooding the petri plates with distilled water. The spore suspension was filtered through four layers of gauze to remove mycelial fragments, and spore concentrations were determined with a hemocytometer under a microscope.

Appressorium formation assay. Spores were placed on a piece of cellophane and were cultured for $10 \mathrm{~h}$ at $22^{\circ} \mathrm{C}$. The cellophane paper was then transferred to a glass slide and was observed under a Leica DM 2500 microscope (Leica).

Epidermal penetration assay. Onion epidermis was harvested and placed on water agar plates and spores were, subsequently, placed on the onion epidermis. The petri plates containing the onion epidermis and spores were then placed in a chamber with high humidity at $22^{\circ} \mathrm{C}$ for $24 \mathrm{~h}$. Infection structures and penetration were then observed and were counted under a Leica DM 2500 microscope after staining the hyphae with lactophenol blue.

\section{Virulence assays.}

Spores were harvested from PDA plates and the resulting spore concentration was determined as previously described. The spore (conidia) concentration was adjusted to $1 \times 10^{4}$ spores per milliliter and $10 \mu \mathrm{l}$ of the conidial suspension was inoculated into wounds of apple or other fruit. Four wounds were made with a sterile nail on each fruit, one for the WT and one for each of three mutant strains. Lesion diameters were measured at various time points over a range of 36 to $120 \mathrm{~h}$, depending on the fruit type being monitored. Alternatively, for the leaf infection assays, the conidial suspension was adjusted to $2 \times 10^{5}$ spores per milliliater with PDB. Leaves were inoculated with $10 \mu \mathrm{l}$ of the spore suspension and were then placed into moist chambers at $22^{\circ} \mathrm{C}$. The plates were monitored for lesion formation at various time points ranging from 48 to $96 \mathrm{~h}$. Three biological repeats were performed in each experiment.

\section{ACKNOWLEDGMENTS}

The authors thank P. Tudzynski (Westfaelische Wilhelms-Universitaet Muenster, Germany) and J. van Kan (Wageningen Agricultural University, The Netherlands) for kindly providing the B. cinerea haploid strain B05.10 and the pLOB7 plasmid, respectively. This work was supported by the National Natural Science Foundation of China (grant numbers 31530057 and 31172004). The funding agency had no role in the study design, data collection and analysis, decision to publish, or preparation of the manuscript.

\section{LITERATURE CITED}

Adams, J., Kelso, R., and Cooley, L. 2000. The kelch repeat superfamily of proteins: Propellers of cell function. Trends Cell Biol. 10:17-24.

Adams, M. J., Ellis, G. H., Gover, S., Naylor, C. E., and Phillips, C. 1994. Crystallographic study of coenzyme, coenzyme analogue and substrate binding in 6-phosphogluconate dehydrogenase: Implications for NADP specificity and the enzyme mechanism. Structure 2:651-668.

Aguirre, J., Ríos-Momberg, M., Hewitt, D., and Hansberg, W. 2005. Reactive oxygen species and development in microbial eukaryotes. Trends Microbiol. 13:111-118.

Ahn, I. P., Kim, S., and Lee, Y. H. 2005. Vitamin B1 functions as an activator of plant disease resistance. Plant Physiol. 138:1505-1515.

Amselem, J., Cuomo, C. A., van Kan, J. A., Viaud, M., Benito, E. P., Couloux, A., Coutinho, P. M., de Vries, R. P., Dyer, P. S., Fillinger, S., Fournier, E., Gout, L., Hahn, M., Kohn, L., Lapalu, N., Plummer, K. M., Pradier, J. M., Quévillon, E., Sharon, A., Simon, A., ten Have, A., Tudzynski, B., Tudzynski, P., Wincker, P., Andrew, M., Anthouard, V., Beever, R. E., Beffa, R., Benoit, I., Bouzid, O., Brault, B., Chen, Z., Choquer, M., Collémare, J., Cotton, P., Danchin, E. G., Da Silva, C., Gautier, A., Giraud, C., Giraud, T., Gonzalez, C., Grossetete, S., Güldener, U., Henrissat, B., Howlett, B. J., Kodira, C., Kretschmer, M., Lappartient, A., Leroch, M., Levis, C., Mauceli, E., Neuvéglise, C., Oeser, B., Pearson, M., Poulain, J., Poussereau, N., Quesneville, H., Rascle, C., Schumacher, J., Ségurens, B., Sexton, A., Silva, E., Sirven, C., Soanes, D. M., Talbot, N. J., Templeton, M., Yandava, C., Yarden, O., Zeng, Q., Rollins, J. A., Lebrun, M. H., and Dickman, M. 2011. Genomic analysis of the necrotrophic fungal pathogens Sclerotinia sclerotiorum and Botrytis cinerea. PLoS Genet. 7:e1002230.

An, B., Li, B., Qin, G., and Tian, S. 2015. Function of small GTPase Rho3 in regulating growth, conidiation and virulence of Botrytis cinerea. Fungal Genet. Biol. 75:46-55.

An, B., Li, B. Q., Li, H., Zhang, Z. Q., Qin, G. Z., and Tian, S. P. 2016. Aquaporin8 regulates cellular development and reactive oxygen species production, a critical component of virulence in Botrytis cinerea. New Phytol. 209:1668-1680.

Banki, K., Hutter, E., Colombo, E., Gonchoroff, N. J., and Perl, A. 1996. Glutathione levels and sensitivity to apoptosis are regulated by changes in transaldolase expression. J. Biol. Chem. 271:32994-33001. 
Belyaeva, O. V., Korkina, O. V., Stetsenko, A. V., and Kedishvili, N. Y. 2008. Human retinol dehydrogenase 13 (RDH13) is a mitochondrial short-chain dehydrogenase/reductase with a retinaldehyde reductase activity. FEBS J. 275:138-147.

Cano-Domínguez, N., Alvarez-Delfín, K., Hansberg, W., and Aguirre, J. 2008. NADPH oxidases NOX-1 and NOX-2 require the regulatory subunit NOR-1 to control cell differentiation and growth in Neurospora crassa. Eukaryot. Cell 7:1352-1361.

Caraux, G., and Pinloche, S. 2005. PermutMatrix: A graphical environment to arrange gene expression profiles in optimal linear order. Bioinformatics 21:1280-1281.

Chen, C., and Dickman, M. B. 2005. Proline suppresses apoptosis in the fungal pathogen Colletotrichum trifolii. Proc. Natl. Acad. Sci. U.S.A. 102:3459-3464.

Chen, F., Pandey, D., Chadli, A., Catravas, J. D., Chen, T., and Fulton, D. J. 2011. Hsp90 regulates NADPH oxidase activity and is necessary for superoxide but not hydrogen peroxide production. Antioxid. Redox Signal. 14:2107-2119.

Choquer, M., Fournier, E., Kunz, C., Levis, C., Pradier, J. M., Simon, A., and Viaud, M. 2007. Botrytis cinerea virulence factors: New insights into a necrotrophic and polyphageous pathogen. FEMS Microbiol. Lett. 277: $1-10$.

Colombo, E., Banki, K., Tatum, A. H., Daucher, J., Ferrante, P., Murray, R. S., Phillips, P. E., and Perl, A. 1997. Comparative analysis of antibody and cell-mediated autoimmunity to transaldolase and myelin basic protein in patients with multiple sclerosis. J. Clin. Invest. 99:1238-1250.

Diekmann, D., Abo, A., Johnston, C., Segal, A. W., and Hall, A. 1994 Interaction of Rac with $\mathrm{p} 67^{\text {phox }}$ and regulation of phagocytic NADPH oxidase activity. Science 265:531-533.

Egan, M. J., Wang, Z. Y., Jones, M. A., Smirnoff, N., and Talbot, N. J. 2007. Generation of reactive oxygen species by fungal NADPH oxidases is required for rice blast disease. Proc. Natl. Acad. Sci. U.S.A. 104: $11772-11777$.

Fernández-Acero, F. J., Jorge, I., Calvo, E., Vallejo, I., Carbú, M., Camafeita, E., López, J. A., Cantoral, J. M., and Jorrín, J. 2006. Twodimensional electrophoresis protein profile of the phytopathogenic fungus Botrytis cinerea. Proteomics 6 (Suppl 1):S88-S96.

Gerke, J., Bayram, O., and Braus, G. H. 2012. Fungal S-adenosylmethionine synthetase and the control of development and secondary metabolism in Aspergillus nidulans. Fungal Genet. Biol. 49:443-454.

González-Rodríguez, V. E., Liñeiro, E., Colby, T., Harzen, A., Garrido, C., Cantoral, J. M., Schmidt, J., and Fernández-Acero, F. J. 2015. Proteomic profiling of Botrytis cinerea conidial germination. Arch. Microbiol. 197: 117-133.

Hackenberg, T., Juul, T., Auzina, A., Gwizdz, S., Malolepszy, A., Van Der Kelen, K., Dam, S., Bressendorff, S., Lorentzen, A., Roepstorff, P., Lehmann Nielsen, K., Jørgensen, J. E., Hofius, D., Van Breusegem, F., Petersen, M., and Andersen, S. U. 2013. Catalase and NO CATALASE ACTIVITY1 promote autophagy-dependent cell death in Arabidopsis. Plant Cell 25:4616-4626.

Hondorp, E. R., and Matthews, R. G. 2004. Oxidative stress inactivates cobalamin-independent methionine synthase (MetE) in Escherichia coli. PLoS Biol. 2:e336.

Kang, M. Y., Kim, H. B., Piao, C., Lee, K. H., Hyun, J. W., Chang, I. Y., and You, H. J. 2013. The critical role of catalase in prooxidant and antioxidant function of p53. Cell Death Differ. 20:117-129.

Kars, I., McCalman, M., Wagemakers, L., and Van Kan, J. A. 2005. Functional analysis of Botrytis cinerea pectin methylesterase genes by PCR-based targeted mutagenesis: Bcpme 1 and Bcpme 2 are dispensable for virulence of strain B05.10. Mol. Plant Pathol. 6:641-652.

Kayano, Y., Tanaka, A., Akano, F., Scott, B., and Takemoto, D. 2013. Differential roles of NADPH oxidases and associated regulators in polarized growth, conidiation and hyphal fusion in the symbiotic fungus Epichloë festucae. Fungal Genet. Biol. 56:87-97.

Kowalska, E., Kujda, M., Wolak, N., and Kozik, A. 2012. Altered expression and activities of enzymes involved in thiamine diphosphate biosynthesis in Saccharomyces cerevisiae under oxidative and osmotic stress. FEMS Yeast Res. 12:534-546.

Lambeth, J. D., and Neish, A. S. 2014. Nox enzymes and new thinking on reactive oxygen: A double-edged sword revisited. Annu. Rev. Pathol. 9:119-145.

Lara-Ortíz, T., Riveros-Rosas, H., and Aguirre, J. 2003. Reactive oxygen species generated by microbial NADPH oxidase NoxA regulate sexual development in Aspergillus nidulans. Mol. Microbiol. 50:1241-1255.

Li, B. Q., Wang, W. H., Zong, Y. Y., Qin, G. Z., and Tian, S. P. 2012 Exploring pathogenic mechanisms of Botrytis cinerea secretome under different ambient $\mathrm{pH}$ based on comparative proteomic analysis. J. Proteome Res. 11:4249-4260.
Livak, K. J., and Schmittgen, T. D. 2001. Analysis of relative gene expression data using real-time quantitative PCR and the $\left.2^{(-\Delta \Delta} \mathrm{C}(\mathrm{T})\right)$ Method. Methods 25:402-408.

Malagnac, F., Lalucque, H., Lepère, G., and Silar, P. 2004. Two NADPH oxidase isoforms are required for sexual reproduction and ascospore germination in the filamentous fungus Podospora anserina. Fungal Genet. Biol. 41:982-997.

Marino, D., Andrio, E., Danchin, E. G., Oger, E., Gucciardo, S., Lambert, A. Puppo, A., and Pauly, N. 2011. A Medicago truncatula NADPH oxidase is involved in symbiotic nodule functioning. New Phytol. 189:580-592.

Matsuura, K., and Takagi, H. 2005. Vacuolar functions are involved in stress-protective effect of intracellular proline in Saccharomyces cerevisiae. J. Biosci. Bioeng. 100:538-544.

Matysik, J., Bhalu, B., and Mohanty, P. 2002. Molecular mechanisms of quenching of reactive oxygen species by proline under stress in plants. Curr. Sci. 82:525-532.

Morita, Y., Nakamori, S., and Takagi, H. 2002. Effect of proline and arginine metabolism on freezing stress of Saccharomyces cerevisiae. J. Biosci. Bioeng. 94:390-394.

Morita, Y., Nakamori, S., and Takagi, H. 2003. L-proline accumulation and freeze tolerance of Saccharomyces cerevisiae are caused by mutation in the PRO1 gene encoding $\gamma$-glutamyl kinase. Appl. Environ. Microbiol 69:212-219.

Muller, F. L., Liu, Y., and Van Remmen, H. 2004. Complex III releases superoxide to both sides of the inner mitochondrial membrane. J. Biol. Chem. 279:49064-49073.

Müller, K., Carstens, A. C., Linkies, A., Torres, M. A., and LeubnerMetzger, G. 2009. The NADPH-oxidase AtrbohB plays a role in Arabidopsis seed after-ripening. New Phytol. 184:885-897.

Niforou, K., Cheimonidou, C., and Trougakos, I. P. 2014. Molecular chaperones and proteostasis regulation during redox imbalance. Redox Biol. 2:323-332.

Pradet-Balade, B., Boulmé, F., Beug, H., Müllner, E. W., and Garcia-Sanz, J. A. 2001. Translation control: Bridging the gap between genomics and proteomics? Trends Biochem. Sci. 26:225-229.

Qin, G., Liu, J., Cao, B., Li, B., and Tian, S. 2011. Hydrogen peroxide acts on sensitive mitochondrial proteins to induce death of a fungal pathogen revealed by proteomic analysis. PLoS One 6:e21945.

Qin, G., Tian, S., Chan, Z., and Li, B. 2007. Crucial role of antioxidant proteins and hydrolytic enzymes in pathogenicity of Penicillium expansum: Analysis based on proteomics approach. Mol. Cell. Proteomics 6:425-438.

Reeves, E. P., Lu, H., Jacobs, H. L., Messina, C. G., Bolsover, S., Gabella, G., Potma, E. O., Warley, A., Roes, J., and Segal, A. W. 2002. Killing activity of neutrophils is mediated through activation of proteases by $\mathrm{K}^{+}$ flux. Nature 416:291-297.

Rhee, S. G., Chae, H. Z., and Kim, K. 2005. Peroxiredoxins: A historical overview and speculative preview of novel mechanisms and emerging concepts in cell signaling. Free Radic. Biol. Med. 38:1543-1552.

Rolke, Y., Liu, S., Quidde, T., Williamson, B., Schouten, A., Weltring, K. M., Siewers, V., Tenberge, K. B., Tudzynski, B., and Tudzynski, P 2004. Functional analysis of $\mathrm{H}_{2} \mathrm{O}_{2}$-generating systems in Botrytis cinerea: The major $\mathrm{Cu}-\mathrm{Zn}$-superoxide dismutase (BCSOD1) contributes to virulence on French bean, whereas a glucose oxidase (BCGOD1) is dispensable. Mol. Plant Pathol. 5:17-27.

Ryder, L. S., Dagdas, Y. F., Mentlak, T. A., Kershaw, M. J., Thornton, C. R., Schuster, M., Chen, J., Wang, Z., and Talbot, N. J. 2013. NADPH oxidases regulate septin-mediated cytoskeletal remodeling during plant infection by the rice blast fungus. Proc. Natl. Acad. Sci. U.S.A. 110: 3179-3184.

Sadler, J. E. 1998. Biochemistry and genetics of von Willebrand factor. Annu. Rev. Biochem. 67:395-424.

Schumacher, J. 2012. Tools for Botrytis cinerea: New expression vectors make the gray mold fungus more accessible to cell biology approaches. Fungal Genet. Biol. 49:483-497.

Scott, B., and Eaton, C. J. 2008. Role of reactive oxygen species in fungal cellular differentiations. Curr. Opin. Microbiol. 11:488-493.

Segmüller, N., Kokkelink, L., Giesbert, S., Odinius, D., van Kan, J., and Tudzynski, P. 2008. NADPH oxidases are involved in differentiation and pathogenicity in Botrytis cinerea. Mol. Plant-Microbe Interact 21: 808-819.

Semighini, C. P., and Harris, S. D. 2008. Regulation of apical dominance in Aspergillus nidulans hyphae by reactive oxygen species. Genetics 179: 1919-1932.

Siegmund, U., Marschall, R., and Tudzynski, P. 2015. BcNoxD, a putative ER protein, is a new component of the NADPH oxidase complex in Botrytis cinerea. Mol. Microbiol. 95:988-1005. 
Siewers, V., Viaud, M., Jimenez-Teja, D., Collado, I. G., Gronover, C. S., Pradier, J. M., Tudzynski, B., and Tudzynski, P. 2005. Functional analysis of the cytochrome P450 monooxygenase gene bcbot1 of Botrytis cinerea indicates that botrydial is a strain-specific virulence factor. Mol. Plant-Microbe Interact 18:602-612.

Stanton, R. C. 2012. Glucose-6-phosphate dehydrogenase, NADPH, and cell survival. IUBMB Life 64:362-369.

Suzuki, N., Miller, G., Morales, J., Shulaev, V., Torres, M. A., and Mittler, R. 2011. Respiratory burst oxidases: The engines of ROS signaling. Curr. Opin. Plant Biol. 14:691-699.

Takemoto, D., Kamakura, S., Saikia, S., Becker, Y., Wrenn, R., Tanaka, A., Sumimoto, H., and Scott, B. 2011. Polarity proteins Bem1 and Cdc24 are components of the filamentous fungal NADPH oxidase complex. Proc. Natl. Acad. Sci. U.S.A. 108:2861-2866.

Takemoto, D., Tanaka, A., and Scott, B. 2007. NADPH oxidases in fungi: Diverse roles of reactive oxygen species in fungal cellular differentiation. Fungal Genet. Biol. 44:1065-1076.

Terao, Y., Nakamori, S., and Takagi, H. 2003. Gene dosage effect of L-proline biosynthetic enzymes on L-proline accumulation and freeze tolerance in Saccharomyces cerevisiae. Appl. Environ. Microbiol. 69:6527-6532.

Tian, S., Qin, G., and Li, B. 2013. Reactive oxygen species involved in regulating fruit senescence and fungal pathogenicity. Plant Mol. Biol. 82:593-602.

Torres, M. A., Dangl, J. L., and Jones, J. D. G. 2002. Arabidopsis gp91phox homologues AtrbohD and AtrbohF are required for accumulation of reactive oxygen intermediates in the plant defense response. Proc. Natl. Acad. Sci. U.S.A. 99:517-522.

Valette-Collet, O., Cimerman, A., Reignault, P., Levis, C., and Boccara, M. 2003. Disruption of Botrytis cinerea pectin methylesterase gene Bcpme1 reduces virulence on several host plants. Mol. Plant-Microbe Interact 16: 360-367.

Verhoeven, N. M., Huck, J. H. J., Roos, B., Struys, E. A., Salomons, G. S., Douwes, A. C., van der Knaap, M. S., and Jakobs, C. 2001. Transaldolase deficiency: Liver cirrhosis associated with a new inborn error in the pentose phosphate pathway. Am. J. Hum. Genet. 68:1086-1092.

Wang, J. W., Groeneveld, D. J., Cosemans, G., Dirven, R. J., Valentijn, K. M., Voorberg, J., Reitsma, P. H., and Eikenboom, J. 2012. Biogenesis of Weibel-Palade bodies in von Willebrand's disease variants with impaired von Willebrand factor intrachain or interchain disulfide bond formation. Haematologica 97:859-866.

Weiberg, A., Wang, M., Lin, F. M., Zhao, H., Zhang, Z., Kaloshian, I., Huang, H. D., and Jin, H. 2013. Fungal small RNAs suppress plant immunity by hijacking host RNA interference pathways. Science 342: 118-123.

Zhang, Z., Qin, G., Li, B., and Tian, S. 2014. Knocking out Bcsas1 in Botrytis cinerea impacts growth, development, and secretion of extracellular proteins, which decreases virulence. Mol. Plant-Microbe Interact 27:590-600.

\section{AUTHOR-RECOMMENDED INTERNET RESOURCES}

Blast2GO homepage: https://www.blast2go.com/home

EnsemblFungi Botrytis cinerea genome database:

http://fungi.ensembl.org/Botrytis_cinerea/Info/Index

Matrix Science Mascot MS/MS Ions web site: www.matrixscience.com

NCBI nonredundant database: http://www.ncbi.nlm.nih. gov 